\title{
25. MAJOR AND TRACE ELEMENT CHEMISTRY OF BASALTS FROM HOLES 417D AND 418A, DEEP SEA DRILLING PROJECT LEGS 51-53
}

\author{
R. Emmermann and H. Puchelt, Institut für Petrographie und Geochemie der Universität Karlsruhe (T.H.), \\ Kaiserstr. 12, D-7500 Karlsruhe, FRG
}

\begin{abstract}
Ninety-six samples of basalts recovered from Holes 417D and 418A, DSDP Legs $51-53$, were analyzed by AAS, INAA, XRF, and wetchemical methods for all major components and 22 trace elements. According to the data obtained, the basalts are relatively fresh and can be classified as low-K tholeiites. They typically show very low concentrations of LIL elements and are strongly depleted in LREE. Intrahole and interhole variation in the overall chemistry is rather limited. The basalts are derived from slightly evolved magmas, which originated from a predepleted peridotitic mantle source. The existing minor differences in average composition between Hole 417D and 418A basalts are due to a different degree of low-pressure fractionation.
\end{abstract}

\section{INTRODUCTION}

The IPOD-I drilling program in the Atlantic was completed by a joint attempt on three consecutive legs (Legs 51, 52 , and 53) to penetrate very deeply (at least $1000 \mathrm{~m}$ ) into oceanic Layer 2. For this purpose, a site was selected at about $25^{\circ} \mathrm{N}$ and $68^{\circ} \mathrm{W}$, located at the southern end of the Bermuda Rise, within the $M O$ magnetic anomaly, and with an inferred age of approximately 109 m.y.B.P. It was envisioned that because of aging and secondary alteration, old oceanic basement would provide favorable conditions for deep penetration with high recovery. Even if the original objective could not be reached for technical problems, the drilling results of these three legs are very impressive.

Altogether, four holes have been drilled, two at Site 417 (Holes 417A and 417D) and two at Site 418 (Holes 418A and 418B), about four kilometers apart (see Figure 1). These holes are characterized as follows: (1) Hole 417A, a single-bit pilot hole, revealed a 209-meter section of highly altered basalts overlain by 208 meters of sediments. (2) Hole 417D (about $450 \mathrm{~m}$ to the west), a multiple re-entry hole, penetrated a sediment cover of 343 meters and drilled a 365.5-meter section of comparatively fresh basalts. (3) Hole $418 \mathrm{~A}$, the second deep penetration attempt, reached a sub-bottom depth of 868 meters, 544 meters of which were in only slightly altered basalts. (4) Hole 418B (some $130 \mathrm{~m}$ to the north of Hole 418A) was drilled to basement, to core the sediment column $(320 \mathrm{~m})$ at this site. Seven meters of basalt were recovered from this single-bit hole.

Due to the exceptionally high rate of basement recovery (which averaged over 70\%), Holes 417D and 418A represent the first complete intervals taken from the upper part of Layer 2 and provide a very detailed glimpse into the structure and composition of the oceanic sea floor produced during an early stage of the evolution of the Atlantic. Substantial evidence indicates that the basalts recovered from these holes are products of a Cretaceous mid-ocean ridge vol- canism and were generated at the ancestral Mid-Atlantic Ridge at about $18^{\circ} \mathrm{N}$. Because of the unexpected freshness of these rocks, unequivocal statements regarding their original composition can be made, which may contribute to solving some outstanding petrogenetic questions. Among these are:

1) What is the chemical nature of ocean-floor basalts erupted during early stages in the development of the oceans?

2) What is their compositional variability?

3) How do they compare to modern ocean-ridge basalts?

4) What genetic conclusion can be drawn from the data obtained?

\section{GENERAL CHARACTERISTICS OF HOLE 417D AND 418A BASALTS}

Before discussing our chemical data obtained in Hole 417D and 418A basalts, a brief summary of the pertinent shipboard results obtained on these rocks will be given (Donnelly et al., 1977; Bryan, Robinson, et al., 1977; Flower, Salisbury, et al., 1977). The basement section drilled in both holes is dominated by pillow basalts, which are sometimes intimately associated with broken-pillow breccia and make up about 70 per cent of the entire profile. Pillow sequences, including pillow breccia, range in thickness from 5 to 120 meters, and are interlayered by massive basalt flows, varying in size between 4 to 25 meters. In the lowermost part of both holes, several small dikes occur (all $<30 \mathrm{~cm}$ wide) which cross-cut massive basalts at high angles and are similar in composition to flows higher in the profile.

Largely on the basis of lithology and phenocryst assemblages, 14 major stratigraphic units have been recognized in Hole 417D and 16 units in Hole 418A. Within the Hole 418A basement section, evidence has been found of 6 tectonic rotations, 5 magnetic field reversals, and 15 distinct 


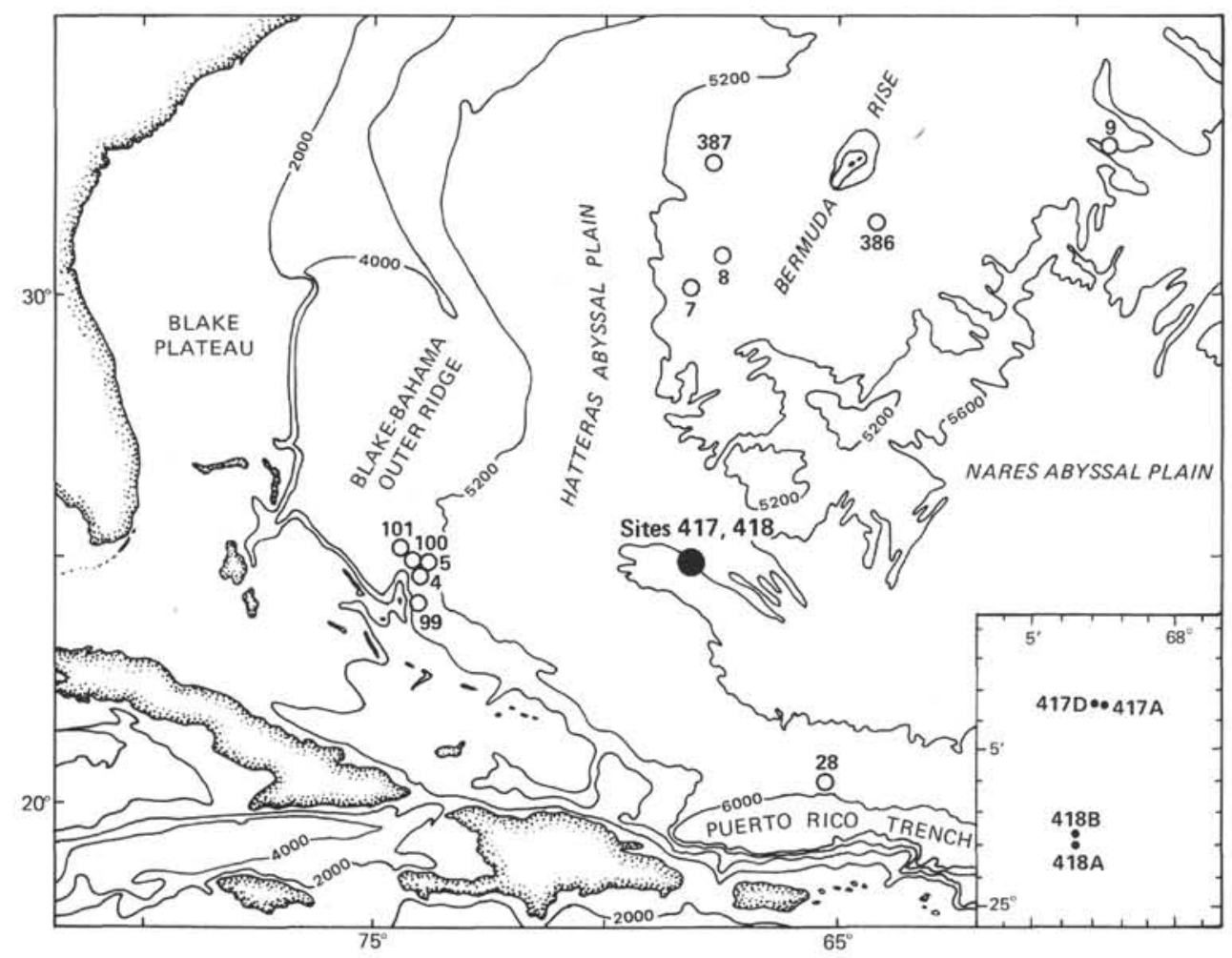

Figure 1. Location map for DSDP Sites 417 and 418.

eruptive events. Nine major time breaks were identified. It was estimated that the volcanic pile cored could have been generated within about 100,000 years.

Mineralogically, the basalts recovered from Holes 417D and $418 \mathrm{~A}$ are very similar. Nearly all basaltic lithologies are porphyritic and contain between 3 and 15 per cent phenocrysts. Plagioclase is always the dominant phenocryst, often in five to ten times greater abundance than the other phenocryst phases. Among those are olivine, which commonly forms $<1$ per cent of the rock, \pm clinopyroxene, occurring preferentially in chemically more-evolved basalts, and spinel, which is a minor but ubiquitous phase in distinct stratigraphic units (e.g., 6 b in Hole 418A). The most widespread phenocryst assemblage among Hole $418 \mathrm{~A}$ basalts is plagioclase plus olivine; whereas in Hole 417D, "three-phenocryst basalts" (plg $+\mathrm{ol}+\mathrm{cpx})$ predominate.

In contrast to the extremely altered basalts drilled in Hole 417A, basalts from Holes 417D and 418A are remarkably fresh. Their slight alteration is very similar in both holes and, most probably, has been caused by low-temperature interaction of rock with sea water. The main secondary minerals are smectite, calcite, pyrite, and zeolites, which commonly occur in veins and as vesicle fillings, but also replace olivine as well as interstitial groundmass. Late-stage oxidation is localized along cracks and appears to postdate the pervasive alteration.

During Legs 51 to 53 , XRF analyses of basalts were performed onboard Glomar Challenger. From Hole 417D, a total of 66 and, from Hole 418A, a total of 157 samples were analyzed for their major oxides (excluding $\mathrm{Na}_{2} \mathrm{O}$, $\mathrm{MnO}$, and $\mathrm{P}_{2} \mathrm{O}_{5}$ ); additionally, $\mathrm{H}_{2} \mathrm{O}^{+}$and $\mathrm{CO}_{2}$ were determined using a $\mathrm{CHN}$ analyzer. According to the data obtained, basalts from both holes are very similar in composition and show a rather limited range of variation. All types recovered can be classified as low- $\mathrm{K}$ olivine tholeiites. Analyses of 14 "fresh" Leg 52 samples from Hole 417D (corrected for carbonate contamination, with $\mathrm{K}_{2} \mathrm{O}<0.20 \%$ and $\mathrm{H}_{2} \mathrm{O}^{+}<1.25 \%$, and normalized to dry weight, assuming $2.5 \mathrm{wt} . \%$ for $\mathrm{Na}_{2} \mathrm{O}$ ) yield the following average composition: $\mathrm{SiO}_{2}=50.0, \mathrm{TiO}_{2}=1.45, \mathrm{Al}_{2} \mathrm{O}_{3}=$ $16.0, \mathrm{FeO}$ (total iron) $=10.0, \mathrm{MgO}=7.0, \mathrm{CaO}=12.5$, and $\mathrm{K}_{2} \mathrm{O}=0.02$. The respective values found for 24 "fresh" basalts from Units 1 through 6 of Hole 418A were: $\mathrm{SiO}_{2}=50.2, \mathrm{TiO}_{2}=1.15, \mathrm{Al}_{2} \mathrm{O}_{3}=16.8, \mathrm{FeO}=9.1$, $\mathrm{MgO}=7.0, \mathrm{CaO}=12.9$, and $\mathrm{K}_{2} \mathrm{O}=0.05$.

Preliminary investigations on the chemical effects of alteration on whole-rock analysis showed that both $\mathrm{K}_{2} \mathrm{O}$ and $\mathrm{H}_{2} \mathrm{O}^{+}$increase significantly with increasing degree of alteration and, thus, can be regarded as very sensitive indicators of the "freshness" of the basalts. Additionally, it was demonstrated that increased amounts of $\mathrm{CO}_{2}$ always correlate with higher concentrations in $\mathrm{CaO}$, suggesting introduction of secondary calcite to the rocks.

\section{CHEMICAL INVESTIGATIONS}

Based on our XRF shipboard analyses, we selected the freshest material possible for this investigation, taking care that all major lithologic units of both holes are duly represented. As criteria for freshness, the concentrations of $\mathrm{K}_{2} \mathrm{O}, \mathrm{H}_{2} \mathrm{O}^{+}$, and $\mathrm{CO}_{2}$ were used according to the arbitrary limits defined in the shipboard reports. Samples were re- 
garded as being altered if one or more of the following criteria were met: $\mathrm{K}_{2} \mathrm{O}>0.25$ per cent, $\mathrm{H}_{2} \mathrm{O}_{+}>1.25$ per unit, and $\mathrm{CO}_{2}>0.15$ per cent.

From the material sampled onboard ship, a total of 96 samples was selected for detailed chemical study: 29 from Hole 417D, and 67 from Hole 418A. All samples were analyzed for their major components including $\mathrm{H}_{2} \mathrm{O}^{+}$and $\mathrm{CO}_{2}$; additionally, 22 trace elements have been determined. The analytical techniques used were: (1) Wet chemical/ photometric/gravimetric for $\mathrm{Si}, \mathrm{Ti}$, total iron, $\mathrm{Fe}^{2+} ; \mathrm{P}$, $\mathrm{H}_{2} \mathrm{O}^{+}$, and $\mathrm{CO}_{2}$. (2) Atomic absorption spectrometry (AAS) for $\mathrm{Al}, \mathrm{Fe}, \mathrm{Mn}, \mathrm{Mg}, \mathrm{Ca}, \mathrm{Na}, \mathrm{K}, \mathrm{Li}, \mathrm{V}, \mathrm{Cr}, \mathrm{Ni}, \mathrm{Cu}, \mathrm{Zn}$, and Sr. (3) Instrumental neutron activation analysis (INAA) for $\mathrm{Fe}, \mathrm{Mn}, \mathrm{Na}, \mathrm{Sc}, \mathrm{Cr}, \mathrm{Co}, \mathrm{Hf}$, and the rare earth elements (REE) La, Ce, Nd, Sm, Eu, Tb, Dy, Yb, and Lu. (4) X-ray fluorescence analysis (XRF) for $\mathrm{Cr}, \mathrm{Ni}, \mathrm{Sr}, \mathrm{Y}$, and $\mathrm{Zr}$. (5) Pyrohydrolysis/ion-sensitive electrode for fluorine.

For the following elements, the concentrations were found to be below the detection limit (d.I. and method used in parentheses): $\mathrm{Rb}(5 \mathrm{ppm}, \mathrm{XRF}) ; \mathrm{Nb}(5 \mathrm{ppm}, \mathrm{XRF}) ; \mathrm{Ba}$ (50 ppm, XRF); Cs (0.7 ppm, INAA); Sb 0.5 ppm, INAA); Ta (0.1 ppm, INAA); Th ( $1 \mathrm{ppm}$, INAA); and U $(0.5 \mathrm{ppm}$, INAA). Analytical details are given in Puchelt et al. (1976).

Precision was tested by replicate measurements of selected samples; accuracy was always checked by carrying reference rocks (such as BCR-1, AGV-1, GSP-1, BM, and $\mathrm{BHVO}$ ) through the whole procedure along with each set of samples. The values obtained by us for the BCR-1 are summarized in Table 1 along with the precision (as $\pm \sigma$ in

TABLE 1

Analytical Results for the BCR-1, Interlaboratory "Standard" A-II-92, and Wedepohl's (1975) Data on Abyssal Oceanic Tholeiites

\begin{tabular}{|c|c|c|c|c|}
\hline \multirow[b]{2}{*}{$\begin{array}{l}\text { Compounds } \\
\text { and Elements }\end{array}$} & \multicolumn{2}{|c|}{ BCR-1 } & \multirow{2}{*}{$\begin{array}{l}\text { Leg } 51-53 \text { "standard" } \\
\text { A-11-92 }\end{array}$} & \multirow{2}{*}{$\begin{array}{c}\text { Tholeiite } \\
\text { Wedepohl (1975) }\end{array}$} \\
\hline & This Work & $\begin{array}{c}\text { Precision } \\
\text { (wt. \%, resp. ppm) }\end{array}$ & & \\
\hline $\mathrm{SiO}_{2}$ & 54.3 & \pm 0.30 & 49.4 & 49.6 \\
\hline $\mathrm{TiO}_{2}$ & 2.31 & \pm 0.03 & 1.77 & 1.43 \\
\hline $\mathrm{Al}_{2} \mathrm{O}_{3}$ & n.d. & \pm 0.25 & 16.0 & 16.0 \\
\hline $\mathrm{Fe}_{2} \mathrm{O}_{3}$ & 3.82 & \pm 0.10 & 2.22 & 1.53 \\
\hline $\mathrm{FeO}$ & 8.63 & \pm 0.15 & 7.71 & 7.80 \\
\hline $\mathrm{MnO}$ & 0.19 & \pm 0.01 & 0.19 & 0.17 \\
\hline $\mathrm{MgO}$ & 3.57 & \pm 0.07 & 7.53 & 7,94 \\
\hline $\mathrm{CaO}$ & 7.03 & \pm 0.10 & 10.7 & 11.2 \\
\hline $\mathrm{Na}_{2} \mathrm{O}$ & 3.33 & \pm 0.05 & 2.98 & 2.76 \\
\hline $\mathrm{K}_{2} \mathrm{O}$ & 1.85 & \pm 0.04 & 0.20 & 0.21 \\
\hline $\mathrm{P}_{2} \mathrm{O}_{5}$ & 0.35 & \pm 0.01 & 0.19 & 0.14 \\
\hline $\mathrm{H}_{2}^{-} \mathrm{O}^{+}$ & 0.82 & \pm 0.05 & 1.00 & no value \\
\hline $\mathrm{CO}_{2}$ & $<0.1$ & \pm 0.10 & 0.12 & no value \\
\hline Li & 12 & \pm 1.0 & 4 & 8 \\
\hline F & 455 & \pm 20 & 200 & no value \\
\hline $\mathrm{Se}$ & 32 & \pm 0.7 & 38 & 52 \\
\hline V & 400 & \pm 15 & 340 & 303 \\
\hline $\mathrm{Cr}_{\mathrm{r}}$ & 18 & \pm 1 & 232 & 298 \\
\hline Co & 37 & \pm 1 & 55 & 37 \\
\hline $\mathrm{Ni}$ & 15 & \pm 1 & 115 & 119 \\
\hline $\mathrm{Cu}$ & 15 & \pm 1 & 65 & 66 \\
\hline $\mathrm{Zn}$ & 114 & \pm 5 & 86 & 84 \\
\hline $\mathrm{Sr}$ & 335 & \pm 10 & 138 & 121 \\
\hline $\mathrm{Zr}$ & 185 & \pm 5 & 105 & 98 \\
\hline $\mathrm{Hf}$ & 4.9 & \pm 0.3 & 2.9 & no value \\
\hline $\mathrm{La}$ & 27.0 & \pm 0.9 & 4.6 & 38 \\
\hline $\mathrm{Ce}$ & 52.0 & \pm 2.5 & 11.0 & 12.4 \\
\hline $\mathrm{Nd}$ & 29.0 & \pm 2.7 & 11.0 & 10.7 \\
\hline Sm & 6.9 & \pm 0.4 & 4.1 & 3.7 \\
\hline Eu & 1.94 & \pm 0.1 & 1.58 & 1.3 \\
\hline $\mathrm{Tb}$ & 1.1 & \pm 0.1 & 1.0 & 0.8 \\
\hline Dy & 6.3 & \pm 0.5 & n.d. & 6.2 \\
\hline $\mathrm{Yb}$ & 3.4 & \pm 0.3 & 3.6 & 2.9 \\
\hline Lu & 0.50 & \pm 0.05 & 0.55 & 0.52 \\
\hline
\end{tabular}

wt.\% or ppm, respectively). This table also includes our data for the interlaboratory Reference Rock A-II-92, which was distributed among the laboratories involved in the analysis of Leg 51-53 basalts.

The major oxide analyses for Hole 417D basalts are presented in Table 2. Each sample is designated according to standard DSDP procedure. The sub-bottom depth is also given along with its stratigraphic position. A close scrutiny of this "raw data" set reveals that only a rather limited variability prevails throughout the analyzed sequence and that the existing differences can be related, at least in part, to varying amounts of $\mathrm{CO}_{2}$ and $\mathrm{H}_{2} \mathrm{O}^{+}$, i.e., to a different degree of alteration. Using the screen defined above, 27 out of the 29 samples analyzed have to be regarded as "altered," 26 specimens would have been so classified on the basis of $\mathrm{H}_{2} \mathrm{O}^{+}$and 25 on the basis of $\mathrm{CO}_{2}$, as compared to only 4 on the basis of $\mathrm{K}_{2} \mathrm{O}$.

To eliminate or at least to reduce the effects due to postmagmatic processes and thus to gain a more reliable basis for discussing primary compositional variations, the "raw data set" was recalculated by applying the following assumptions:

1) $\mathrm{CO}_{2}$ reflects addition of secondary calcite, which simply acts as a dilutent. Consequently, a carbonate correction has been carried out on all samples by subtracting a $\mathrm{CO}_{2}$-equivalent amount of $\mathrm{CaO}$ from the analyzed $\mathrm{CaO}$ values. This procedure seems to be justified from thin-section studies, which reveal that calcite occurs preferentially in veins and vesicles within the rocks. It is also supported by the $\mathrm{CO}_{2}-\mathrm{CaO}$ correlation diagram for all 417D and 418A basalts shown in Figure 2.

2) $\mathrm{H}_{2} \mathrm{O}^{+}$acts essentially as a dilutent without larger effects on the ratios of the other components. This is substantiated, for example, by a comparison of several hundred glass and whole-rock analyses of Leg 37 material, which demonstrated that as much as 3 weight per cent $\mathrm{H}_{2} \mathrm{O}$ can be present in whole-rock analysis without changing the ratios of the other major components (Byerly and Wright, 1977). Thus, a normalization of the major oxides to a "water-free" composition will remove effects due to varying amounts of $\mathrm{H}_{2} \mathrm{O}^{+}$.

3) The analytically obtained values for $\mathrm{Fe}_{2} \mathrm{O}_{3}$ and $\mathrm{FeO}$ do not represent the initial $\mathrm{Fe}^{3+}$ and $\mathrm{Fe}^{2+}$ concentrations, but have been changed to a different degree by late or postmagmatic processes. To overcome this effect, the total iron content measured was redistributed among $\mathrm{Fe}^{2+}$ and $\mathrm{Fe}^{3+}$, assuming a primary ratio of $\mathrm{Fe}^{3+} /\left(\mathrm{Fe}^{2+}+\mathrm{Fe}^{3+}\right)$ of 0.15 .

4) Increased amounts of $\mathrm{K}_{2} \mathrm{O}$ are probably due to smectite formation. No reasonable correction can be applied in this case, because the chemical changes involved are too complex.

The water-free, carbonate, and $\mathrm{Fe}^{2+} / \mathrm{Fe}^{3+}$ corrected analyses of Hole 417D basalts, normalized to 100 per cent, are presented in Table 3 . Table 4 gives a comparison between the averages and the standard deviations of all raw data with the respective values obtained on the "corrected" data set. As was to be expected, scattering of all major components diminished or remains unchanged. This is especially visualized in a frequency distribution plot (Figure 3 ), which reveals a narrow concentration range for all oxides. The apparent bimodal distribution of $\mathrm{TiO}_{2}$ is mainly 
TABLE 2

Major Oxide Compositions of Hole 417D Basalts ("raw data" set)

\begin{tabular}{|c|c|c|c|c|c|c|c|c|c|c|c|c|c|c|c|c|c|}
\hline No. & $\begin{array}{c}\text { Sample } \\
\text { (Interval in } \mathrm{cm} \text { ) }\end{array}$ & $\begin{array}{l}\text { Depth } \\
\text { (m) }\end{array}$ & Unit & $\mathrm{SiO}_{2}$ & $\mathrm{TiO}_{2}$ & $\mathrm{Al}_{2} \mathrm{O}_{3}$ & $\mathrm{Fe}_{2} \mathrm{O}_{3}$ & $\mathrm{FeO}$ & $\mathrm{MgO}$ & $\mathrm{MnO}$ & $\mathrm{CaO}$ & $\mathrm{Na}_{2} \mathrm{O}$ & $\mathrm{K}_{2} \mathrm{O}$ & $\mathrm{P}_{2} \mathrm{O}_{5}$ & $\mathrm{H}_{2} \mathrm{O}^{+}$ & $\mathrm{CO}_{2}$ & Total \\
\hline 1 & $28-5,46-48$ & 382.2 & $1 \mathrm{~b}$ & 45.3 & 1.30 & 15.8 & 5.32 & 4.26 & 5.47 & 0.16 & 14.8 & 2.33 & 0.17 & 0.13 & 2.06 & 3.14 & 100.24 \\
\hline 2 & $33-3,67-69$ & 424.8 & 3 & 49.0 & 1.42 & 16.8 & 4.32 & 5.66 & 6.38 & 0.14 & 12.0 & 2.58 & 0.07 & 0.14 & 1.10 & 0.39 & 100.00 \\
\hline 3 & $39-4,30-32$ & 469.5 & 4 & 45.4 & 1.34 & 16.1 & 5.85 & 4.73 & 5.97 & 0.20 & 13.9 & 2.25 & 0.33 & 0.14 & 1.70 & 2.46 & 100.37 \\
\hline 4 & $41-1,35-37$ & 476.0 & 4 & 45.0 & 1.27 & 16.0 & 6.40 & 3.60 & 6.38 & 0.22 & 13.3 & 2.26 & 0.46 & 0.15 & 2.47 & 2.98 & 100.49 \\
\hline 5 & $43-5,141-143$ & 501.3 & 6 & 47.3 & 1.48 & 16.3 & 5.97 & 5.03 & 6.47 & 0.18 & 11.2 & 2.33 & 0.42 & 0.16 & 1.86 & 1.35 & 100.05 \\
\hline 6 & $44-4,35-38$ & 507.8 & 7 & 48.0 & 1.55 & 15.8 & 5.26 & 5.37 & 6.80 & 0.18 & 12.0 & 2,48 & 0.12 & 0.16 & 1.84 & 0.48 & 100.04 \\
\hline 7 & $48-6,109-111$ & 534.6 & $8 a$ & 47.5 & 1.61 & 16.5 & 5.61 & 4.13 & 6.96 & 0.15 & 11.6 & 2.56 & 0.15 & 0.15 & 1.91 & 1.08 & 99.91 \\
\hline 8 & $48-7,93-95$ & 535.9 & $8 a$ & 48.5 & 1.64 & 17.4 & 5.49 & 3.11 & 6.63 & 0.13 & 11.1 & 2.85 & 0.09 & 0.16 & 1.97 & 0.99 & 100.06 \\
\hline 9 & $50-2,38-40$ & 546.9 & $8 b$ & 49.6 & 1.53 & 15.5 & 4.40 & 4.69 & 6.13 & 0.17 & 12.3 & 2.61 & 0.11 & 0.19 & 1.40 & 1.11 & 99.74 \\
\hline 10 & $52-1,117-119$ & 559.2 & $8 b$ & 46.8 & 1.46 & 15.4 & 4.73 & 4.66 & 6.71 & 0.18 & 13.8 & 2.43 & 0.07 & 0.16 & 1.45 & 2.41 & 100.26 \\
\hline 11 & $53-1,24-26$ & 567.8 & $9 \mathrm{a}$ & 46.9 & 1.51 & 16.4 & 5.37 & 5.47 & 6.70 & 0.21 & 12.4 & 2.38 & 0.11 & 0.17 & 1.55 & 0.91 & 100.08 \\
\hline 12 & $54-6,62-64$ & 585.1 & $9 \mathrm{a}$ & 47.7 & 1.63 & 15.9 & 5.53 & 5.92 & 7.05 & 0.20 & 11.8 & 2.38 & 0.07 & 0.16 & 1.58 & 0.22 & 100.14 \\
\hline 13 & $55-2,95-97$ & 589.0 & $9 \mathrm{a}$ & 46.7 & 1.53 & 14.5 & 4.84 & 6.34 & 7.30 & 0.19 & 13.3 & 2.26 & 0.04 & 0.16 & 1.72 & 1.25 & 100.13 \\
\hline 14 & $57-1,29-31$ & 605.8 & $9 a$ & 47.0 & 1.55 & 16.2 & 5.82 & 5.36 & 6.72 & 0.19 & 12.1 & 2.28 & 0.20 & 0.17 & 1.59 & 0.86 & 100.04 \\
\hline 15 & $57-3,94-96$ & 609.5 & $9 a$ & 48.7 & 1.53 & 18.2 & 5.34 & 3.47 & 7.13 & 0.14 & 10.5 & 2.68 & 0.24 & 0.15 & 1.74 & 0.21 & 100.03 \\
\hline 16 & $59-3,85-87$ & 627.9 & $9 \mathrm{~b}$ & 46.5 & 1.26 & 18.0 & 4.46 & 4.74 & 6.22 & 0.24 & 13.4 & 2.39 & 0.06 & 0.13 & 1.33 & 1.28 & 100.01 \\
\hline 17 & $59-6,33-35$ & 631.8 & $9 b$ & 47.5 & 1.53 & 17.5 & 4.93 & 3.60 & 6.80 & 0.16 & 12.2 & 2.58 & 0.09 & 0.13 & 1.94 & 1.00 & 99.96 \\
\hline 18 & $60-4,19-21$ & 637.7 & $9 c$ & 48.2 & 1.40 & 17.9 & 4.59 & 4.52 & 6.88 & 0.18 & 12.0 & 2.75 & 0.04 & 0.12 & 1.55 & $<0.1$ & 100.13 \\
\hline 19 & $62-4,126-128$ & 647.8 & $9 d$ & 47.3 & 1.38 & 17.5 & 5.19 & 5.43 & 6.20 & 0.19 & 12.2 & 2.31 & 0.04 & 0.13 & 1.49 & 0.66 & 100.02 \\
\hline 20 & $63-2,140-142$ & 653.9 & $9 d$ & 48.4 & 1.42 & 18.5 & 5.06 & 4.63 & 5.97 & 0.17 & 11.5 & 2.44 & 0.04 & 0.13 & 1.61 & 0.22 & 100.09 \\
\hline 21 & $64-4,119-121$ & 665.7 & $10 \mathrm{a}$ & 48.6 & 1.42 & 17.1 & 3.48 & 6.97 & 6.05 & 0.24 & 12.1 & 2.36 & 0.04 & 0.13 & 1.34 & $<0.1$ & 99.83 \\
\hline 22 & $64-5,66-68$ & 666.7 & $10 \mathrm{a}$ & 48.3 & 1.65 & 14.5 & 6.77 & 5.21 & 7.79 & 0.18 & 11.1 & 2.43 & 0.06 & 0.16 & 1.79 & 0.19 & 100.13 \\
\hline 23 & $65-2,117-119$ & 671.7 & $10 \mathrm{~b}$ & 49.1 & 1.48 & 18.0 & 4.53 & 4.12 & 6.04 & 0.20 & 11.9 & 2.80 & 0.27 & 0.13 & 1.53 & 0.33 & 100.43 \\
\hline 24 & $66-3,57-59$ & 681.6 & 11 & 48.8 & 1.44 & 17.5 & 5.50 & 4.50 & 4.98 & 0.14 & 12.7 & 2.53 & 0.05 & 0.14 & 1.77 & 0.25 & 100.30 \\
\hline 25 & $67-1,85-87$ & 687.9 & 12 & 48.6 & 1.36 & 16.9 & 4.43 & 5.83 & 6.87 & 0.18 & 11.8 & 2.43 & 0.04 & 0.13 & 1.31 & 0.33 & 100.21 \\
\hline 26 & $67-5,14-16$ & 693.1 & 12 & 48.6 & 1.33 & 17.4 & 4.73 & 5.59 & 6.55 & 0.17 & 11.7 & 2.31 & 0.04 & 0.13 & 1.50 & 0.10 & 100.15 \\
\hline 27 & $67-6,55-57$ & 695.1 & 13 & 48.6 & 1.38 & 16.8 & 6.00 & 4.23 & 6.88 & 0.16 & 11.3 & 2.43 & 0.08 & 0.17 & 1.70 & 0.41 & 100.14 \\
\hline 28 & $68-5,62-64$ & 702.6 & 13 & 49.5 & 1.50 & 15.3 & 4.39 & 6.65 & 6.90 & 0.18 & 11.7 & 2.39 & 0.09 & 0.17 & 1.20 & 0.15 & 100.12 \\
\hline 29 & $69-1,135-137$ & 707.0 & 13 & 48.6 & 1.33 & 17.5 & 3.87 & 6.44 & 7.13 & 0.17 & 11.5 & 2.19 & 0.07 & 0.13 & 1.15 & $<0.1$ & 100.08 \\
\hline
\end{tabular}

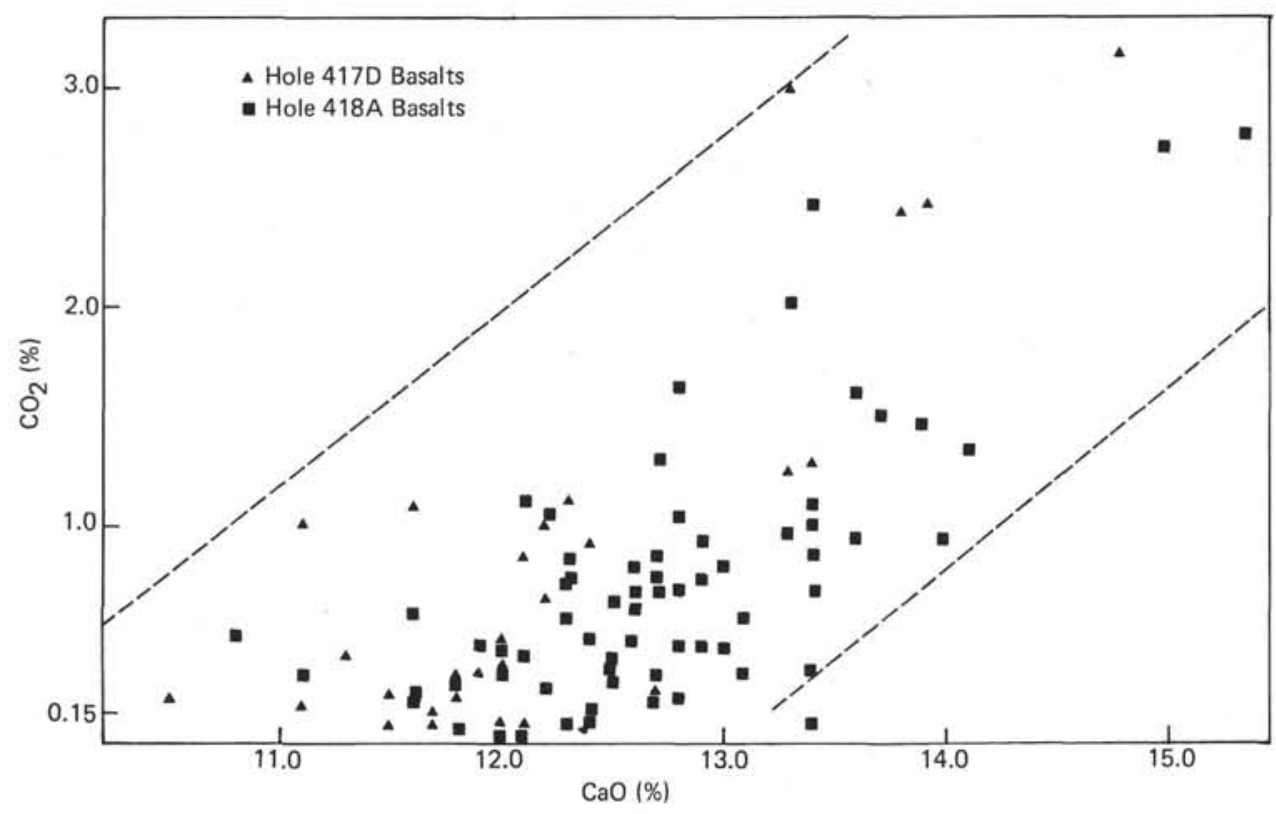

Figure 2. $\mathrm{CO}_{2}-\mathrm{CaO}$ variation diagram for Hole $417 \mathrm{D}$ and $418 \mathrm{~A}$ basalts.

due to the concentration classes chosen and does not point to systematic differences between lithologic units. The skewed curve of $\mathrm{K}_{2} \mathrm{O}$ is due to the very low concentrations and presentation in a linear scale. Values above 0.25 weight per cent are regarded as being caused by alteration.

Altogether, these patterns demonstrate the uniformity of the basalt sequence drilled in Hole 417D. There might be some minor downhole variations which can be related to different stratigraphic units, but the compositional differ- ences are too small to be significant. Thus, as far as the major oxides are concerned, it can be stated that the volcanic pile drilled in Hole 417D was built up by a number of discrete magma eruptions of almost the same major element composition.

The uniformity found for the major components is also reflected in all trace element distributions (Table 5). None of the 22 elements determined can be used to discriminate between different lithologies. Variations between lithologic 
TABLE 3

Recalculated Major Oxide Compositions of Hole 417D Basalts (see text for explanation)

\begin{tabular}{|c|c|c|c|c|c|c|c|c|c|c|c|c|}
\hline No. & $\mathrm{SiO}_{2}$ & $\mathrm{TiO}_{2}$ & $\mathrm{Al}_{2} \mathrm{O}_{3}$ & $\mathrm{Fe}_{2} \mathrm{O}_{3}$ & $\mathrm{FeO}$ & $\mathrm{MgO}$ & $\mathrm{MnO}$ & $\mathrm{CaO}$ & $\mathrm{Na}_{2} \mathrm{O}$ & $\mathrm{K}_{2} \mathrm{O}$ & $\mathrm{P}_{2} \mathrm{O}_{5}$ & $\mathrm{Mg} / \mathrm{Mg}+\mathrm{Fe}^{2+}$ \\
\hline 1 & 49.9 & 1.43 & 17.4 & 1.66 & 8.46 & 6.02 & 0.18 & 12.0 & 2.56 & 0.19 & 0.14 & 0.56 \\
\hline 2 & 50.1 & 1.45 & 17.2 & 1.63 & 8.31 & 6.53 & 0.14 & 11.8 & 2.64 & 0.07 & 0.14 & 0.58 \\
\hline 3 & 48.4 & 1.43 & 17.2 & 1.78 & 9.07 & 6.34 & 0.21 & 12.6 & 2.40 & 0.35 & 0.15 & 0.55 \\
\hline 4 & 49.5 & 1.40 & 17.6 & 1.71 & 8.76 & 7.02 & 0.24 & 10.6 & 2.49 & 0.51 & 0.17 & 0.59 \\
\hline 5 & 49.9 & 1.56 & 17.2 & 1.83 & 9.34 & 6.83 & 0.19 & 10.0 & 2.46 & 0.44 & 0.17 & 0.57 \\
\hline 6 & 49.6 & 1.60 & 16.3 & 1.74 & 8.87 & 7.02 & 0.19 & 11.8 & 2.56 & 0.12 & 0.17 & 0.59 \\
\hline 7 & 49.9 & 1.69 & 17.3 & 1.61 & 8.20 & 7.31 & 0.16 & 10.7 & 2.69 & 0.16 & 0.16 & 0.61 \\
\hline 8 & 50.8 & 1.72 & 18.2 & 1.40 & 7.16 & 6.94 & 0.14 & 10.4 & 2.98 & 0.09 & 0.17 & 0.63 \\
\hline 9 & 51.9 & 1.60 & 16.2 & 1.51 & 7.70 & 6.42 & 0.18 & 11.4 & 2.73 & 0.12 & 0.20 & 0.60 \\
\hline 10 & 50.3 & 1.57 & 16.5 & 1.60 & 8.14 & 7.21 & 0.19 & 11.6 & 2.61 & 0.08 & 0.17 & 0.61 \\
\hline 11 & 48.9 & 1.57 & 16.9 & 1.78 & 9.13 & 6.98 & 0.22 & 11.8 & 2.48 & 0.11 & 0.18 & 0.58 \\
\hline 12 & 48.8 & 1.67 & 16.3 & 1.85 & 9.49 & 7.22 & 0.20 & 11.8 & 2.44 & 0.07 & 0.16 & 0.58 \\
\hline 13 & 49.0 & 1.61 & 15.2 & 1.87 & 9.55 & 7.66 & 0.20 & 12.3 & 2.37 & 0.04 & 0.17 & 0.59 \\
\hline 14 & 48.9 & 1.61 & 16.9 & 1.83 & 9.47 & 7.00 & 0.20 & 11.5 & 2.37 & 0.21 & 0.18 & 0.57 \\
\hline 15 & 50.0 & 1.57 & 18.7 & 1.42 & 7.23 & 7.32 & 0.14 & 10.5 & 2.75 & 0.25 & 0.15 & 0.64 \\
\hline 16 & 48.7 & 1.32 & 18.8 & 1.52 & 7.79 & 6.51 & 0.25 & 12.4 & 2.50 & 0.06 & 0.14 & 0.60 \\
\hline 17 & 49.8 & 1.60 & 18.3 & 1.40 & 7.16 & 7.13 & 0.17 & 11.5 & 2.70 & 0.09 & 0.14 & 0.64 \\
\hline 18 & 49.1 & 1.43 & 18.2 & 1.47 & 7.48 & 7.00 & 0.18 & 12.2 & 2.80 & 0.04 & 0.12 & 0.63 \\
\hline 19 & 48.9 & 1.43 & 18.1 & 1.74 & 8.88 & 6.41 & 0.20 & 11.8 & 2.39 & 0.04 & 0.13 & 0.56 \\
\hline 20 & 49.6 & 1.45 & 18.9 & 1.57 & 7.99 & 6.12 & 0.17 & 11.5 & 2.50 & 0.04 & 0.13 & 0.58 \\
\hline 21 & 49.5 & 1.45 & 17.4 & 1.71 & 8.74 & 6.16 & 0.24 & 12.2 & 2.40 & 0.04 & 0.13 & 0.56 \\
\hline 22 & 49.6 & 1.69 & 14.9 & 1.93 & 9.86 & 7.99 & 0.18 & 11.2 & 2.49 & 0.06 & 0.16 & 0.59 \\
\hline 23 & 50.2 & 1.51 & 18.4 & 1.40 & 7.12 & 6.17 & 0.20 & 11.8 & 2.86 & 0.28 & 0.13 & 0.61 \\
\hline 24 & 50.0 & 1.47 & 17.9 & 1.62 & 9.01 & 5.10 & 0.14 & 12.7 & 2.59 & 0.05 & 0.14 & 0.50 \\
\hline 25 & 49.6 & 1.39 & 17.3 & 1.66 & 8.53 & 7.01 & 0.18 & 11.6 & 2.48 & 0.04 & 0.13 & 0.59 \\
\hline 26 & 49.5 & 1.36 & 17.7 & 1.70 & 8.53 & 6.67 & 0.17 & 11.8 & 2.35 & 0.04 & 0.13 & 0.58 \\
\hline 27 & 50.0 & 1.42 & 17.3 & 1.65 & 8.44 & 7.09 & 0.16 & 11.1 & 2.50 & 0.08 & 0.18 & 0.60 \\
\hline 28 & 50.3 & 1.53 & 15.6 & 1.79 & 9.16 & 7.02 & 0.18 & 11.7 & 2.43 & 0.09 & 0.17 & 0.58 \\
\hline 29 & 49.3 & 1.35 & 17.7 & 1.67 & 8.55 & 7.23 & 0.17 & 11.6 & 2.22 & 0.07 & 0.17 & 0.60 \\
\hline
\end{tabular}

TABLE 4

Average Major Oxide Compositions of Hole 417D and 418A Basalts

\begin{tabular}{|c|c|c|c|c|c|c|c|c|}
\hline \multirow[b]{3}{*}{ Compounds } & \multicolumn{4}{|c|}{ 417D Basalts } & \multicolumn{4}{|c|}{$418 \mathrm{~A}$ Basalts } \\
\hline & \multicolumn{2}{|c|}{ "Raw" Data } & \multicolumn{2}{|c|}{ "Corrected" Data } & \multicolumn{2}{|c|}{ "Raw" Data } & \multicolumn{2}{|c|}{ "Corrected" Data } \\
\hline & $\bar{x}$ & $\pm \delta$ & $\bar{x}$ & $\pm \delta$ & $\bar{x}$ & $\pm \delta$ & $\bar{x}$ & $\pm \delta$ \\
\hline $\mathrm{SiO}_{2}$ & 47.8 & 1.2 & 49.7 & 0.7 & 48.5 & 0.8 & 50.1 & 0.5 \\
\hline $\mathrm{TiO}_{2}$ & 1.46 & 0.11 & 1.51 & 0.11 & 1.25 & 0.16 & 1.27 & 0.14 \\
\hline $\mathrm{Al}_{2} \mathrm{O}_{3}$ & 16.7 & 1.1 & 17.3 & 1.0 & 16.2 & 1.0 & 16.7 & 1.0 \\
\hline $\mathrm{Fe}_{2} \mathrm{O}_{3}$ & 5.11 & 0.75 & 1.66 & 0.15 & 4.29 & 0.66 & 1.59 & 0.11 \\
\hline $\mathrm{FeO}$ & 4.97 & 0.98 & 8.49 & 0.78 & 5.45 & 1.02 & 8.15 & 0.58 \\
\hline $\mathrm{MgO}$ & 6.55 & 0.57 & 6.81 & 0.57 & 7.03 & 0.87 & 7.26 & 0.87 \\
\hline $\mathrm{MnO}$ & 0.18 & 0.03 & 0.19 & 0.03 & 0.17 & 0.03 & 0.18 & 0.03 \\
\hline $\mathrm{CaO}$ & 12.2 & 1.0 & 11.6 & 0.65 & 12.7 & 0.8 & 12.6 & 0.6 \\
\hline $\mathrm{Na}_{2} \mathrm{O}$ & 2.45 & 0.17 & 2.54 & 0.17 & 2.26 & 0.16 & 2.34 & 0.16 \\
\hline $\mathrm{K}_{2} \mathrm{O}$ & 0.09 & 0.05 & 0.09 & 0.05 & 0.07 & 0.04 & 0.07 & 0.04 \\
\hline $\mathrm{P}_{2} \mathrm{O}_{5}$ & 0.15 & 0.02 & 0.15 & 0.02 & 0.12 & 0.03 & 0.13 & 0.03 \\
\hline
\end{tabular}

units do not exceed variations observed within the units. On the basis of this finding, and from trace element characteristics, Hole 417D basalts represent one single magma type.

Among the trace elements analyzed, the REE are of special importance, since they not only offer additional opportunities for basalt characterization, but also provide valuable indicators in modeling basalt genesis. The chondritenormalized REE values are used in Figure 4 to draw the average distribution pattern (heavy line) and to indicate the range of variation (shaded area). For the normalization, the following chondrite values (in ppm) have been used: $\mathrm{La}=0.315, \mathrm{Ce}=0.813, \mathrm{Nd}=0.5966, \mathrm{Sm}=0.1917$, $\mathrm{Eu}=0.07216, \mathrm{~Tb}=0.049, \mathrm{Dy}=0.2075$, and $\mathrm{Lu}=$ 0.03225 (Masuda, 1975). In general, all chondrite-normalized patterns are characterized by a strong depletion of light rare earth elements (LREE) over the heavier ones
(HREE). As a measure of the degree of fractionation of the LREE, the ratio $\mathrm{La} / \mathrm{Sm}$ (normalized to chondrite) is used and is designated the $\mathrm{La} / \mathrm{Sm}$ enrichment factor (e.f.). This value averages 0.47 for Hole $417 \mathrm{D}$ basalts. With respect to the HREE, Hole 417D basalts are almost unfractionated, i.e., they exhibit chondritic patterns. Therefore, the Yb e.f. provides a reasonable measure for the overall enrichment of the HREE. The average Yb e.f. is 15.9; the lowest and highest values obtained are 11.6 and 23.1 , respectively. A characteristic feature in the REE distribution curve of some ocean floor basalts is the occurrence of a positive or negative Eu anomaly (Puchelt and Emmermann, 1977). These anomalies are expressed by a value $\mathrm{Eu}^{*}$, which is the ratio of the normalized Eu value of the sample to the interpolated Eu value obtained when the REE are connected by a smooth distribution curve. For all Hole 417D basalts, Eu* is very close to unity; i.e., Eu does not show an anomalous behavior with respect to the adjacent REE.

Averages for the other trace elements determined are summarized in Table 6 along with the respective standard deviations. Except for $\mathrm{Li}, \mathrm{F}$, and $\mathrm{Hf}$, the relative standard deviation throughout the basalt sequence is $<16$ per cent.

The analytical values obtained for the major components of the 67 basalt samples selected from Hole $418 \mathrm{~A}$ are presented in Table 7; the corrected and recalculated data set is given in Table 8. Additionally, the oxide means and standard deviations computed from both data sets have been summarized in Table 4. Comparison of these values with the respective values of Hole 417D basalts shows that only minor differences exist in the average bulk composition and that the standard deviations are almost identical (except for $\mathrm{MgO}$, which exhibits a somewhat higher scatter in Hole 

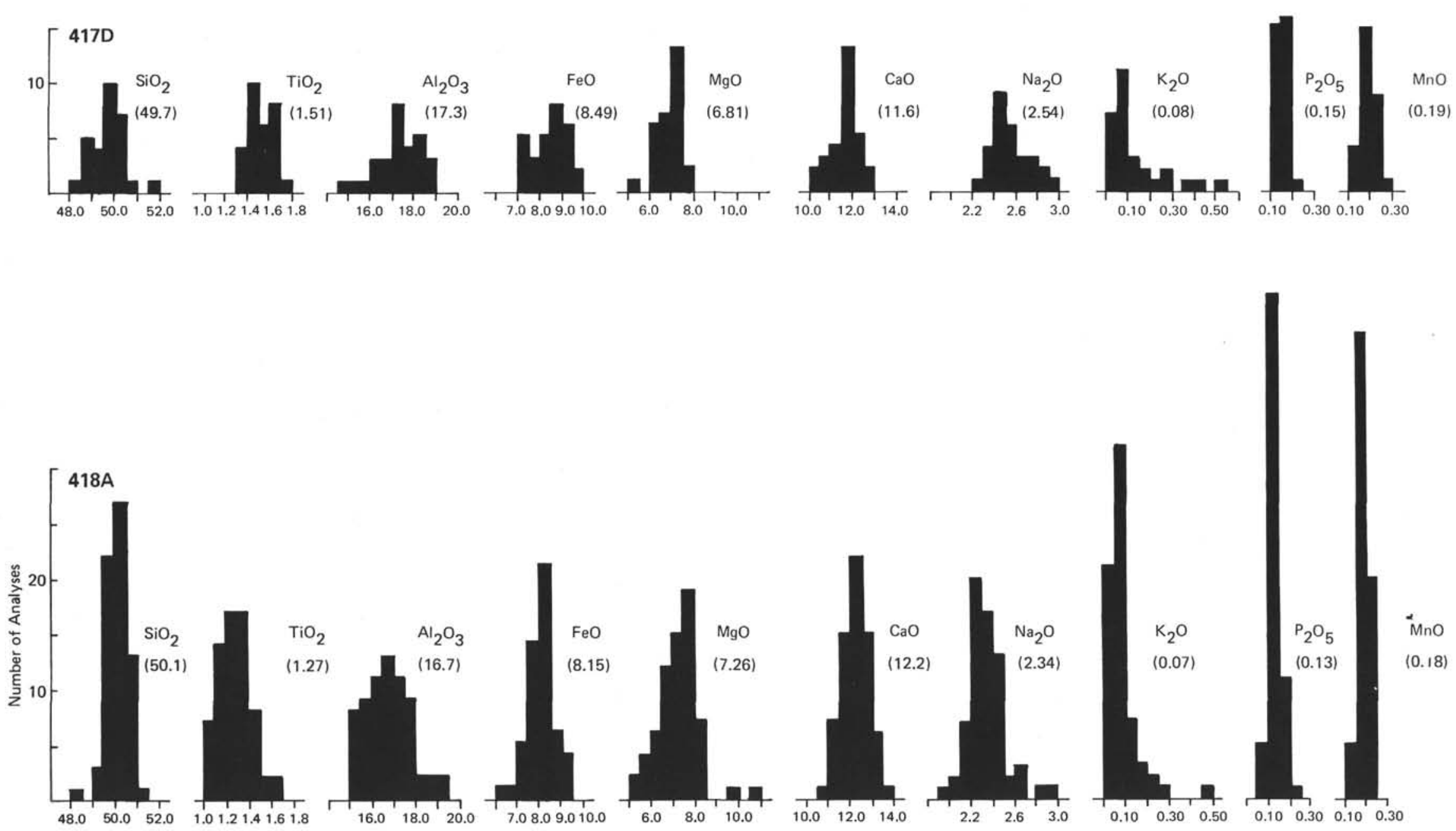

Figure 3. Hole $417 D$ and $418 \mathrm{~A}$ oxide frequencies. 
TABLE 5

Trace Element Chemistry of Hole 417D Basalts

\begin{tabular}{|c|c|c|c|c|c|c|c|c|c|c|c|c|c|c|c|c|c|c|c|c|c|c|c|}
\hline No. & Unit & $\mathbf{L i}$ & F & Sc & V & $\mathrm{Cr}$ & $\mathrm{Co}$ & $\mathrm{Ni}$ & $\mathrm{Cu}$ & $\mathrm{Zn}$ & $\mathrm{Sr}$ & $\mathrm{Y}$ & $\mathrm{Z}_{\mathrm{r}}$ & Hf & $\mathrm{La}$ & $\mathrm{Ce}$ & $\mathrm{Nd}$ & $\mathrm{Sm}$ & $\mathrm{Eu}$ & $\mathrm{Tb}$ & Dy & $\mathrm{Yb}$ & $\mathrm{Lu}$ \\
\hline 1 & $1 b$ & 14 & 330 & 39 & 340 & 265 & 46 & 102 & 104 & 105 & 132 & 32 & 95 & 2.4 & 2.2 & 5.3 & 6.3 & 2.9 & 1.10 & 0.70 & 4.8 & 2.9 & 0.44 \\
\hline 2 & 3 & 9 & n.d. & 42 & 340 & 261 & 46 & 82 & 106 & 104 & 163 & 31 & 89 & 2.9 & 2.8 & 5.5 & 8.7 & 2.7 & 1.06 & 0.75 & 5.6 & 3.3 & 0.53 \\
\hline 3 & 4 & 9 & 190 & 38 & 340 & 263 & 39 & 80 & 86 & 98 & 104 & 22 & 74 & 2.9 & 2.0 & 7.2 & 6.7 & 2.8 & 1.01 & 0.93 & 5.2 & 3.4 & 0.45 \\
\hline 4 & 4 & 11 & n.d. & 38 & 360 & 269 & 40 & 98 & 96 & 115 & 115 & 34 & 74 & 1.7 & 2.9 & 7.3 & 6.9 & 2.5 & 1.11 & 0.71 & 5.5 & 3.3 & 0.47 \\
\hline 5 & 6 & 9 & 240 & 42 & 340 & 242 & 45 & 93 & 94 & 113 & 112 & 33 & 89 & 2.4 & 2.9 & 8.2 & 6.6 & 3.3 & 1.29 & 0.80 & 5.8 & 2.9 & 0.56 \\
\hline 6 & 7 & 9 & n.d. & 44 & 380 & 249 & 50 & 105 & 115 & 112 & 143 & 37 & 88 & 1.5 & 2.4 & 9.5 & 10.0 & 3.5 & 1.45 & 1.00 & 6.2 & 3.7 & 0.49 \\
\hline 7 & $8 a$ & 11 & 200 & 45 & 400 & 257 & 56 & 97 & 114 & 108 & 116 & 26 & 88 & 2.1 & 3.4 & 9.6 & 8.3 & 2.7 & 1.08 & 0.75 & 5.3 & 3.0 & 0.48 \\
\hline 8 & $8 a$ & 14 & n.d. & 46 & 380 & 251 & 63 & 80 & 116 & 110 & 134 & 27 & 103 & 2.9 & 2.2 & 8.0 & 6.7 & 2.8 & 1.12 & 0.50 & 4.9 & 2.9 & 0.49 \\
\hline 9 & $8 b$ & 14 & 320 & 44 & 360 & 241 & 58 & 106 & 120 & 113 & 118 & 36 & 95 & 2.5 & 2.6 & 6. & 7.2 & 3.3 & 1.04 & 0.82 & 5.3 & 3.3 & 0.56 \\
\hline 10 & $8 b$ & 9 & n.d. & 43 & 340 & 310 & 62 & 142 & 114 & 104 & 130 & 30 & 84 & 2.7 & 2.7 & 7.3 & 7.4 & 3.2 & 1.26 & 0.81 & 6.1 & 3.0 & 0.50 \\
\hline 11 & $9 a$ & 9 & 250 & 43 & 380 & 290 & 49 & 93 & 105 & 110 & 104 & 31 & 92 & 2.9 & 1.9 & 8.1 & 7.7 & 3.4 & 1.10 & 0.92 & 6.0 & 3.3 & 0.55 \\
\hline 12 & $9 a$ & 11 & n.d. & 43 & 380 & 242 & 50 & 115 & 104 & 115 & 101 & 18 & 93 & 2. & 3.0 & 12.0 & 11.0 & 3.5 & 1.29 & 0.83 & 7.2 & 4.3 & 0.61 \\
\hline 13 & $9 a$ & 13 & 270 & 42 & 360 & 252 & 45 & 90 & 98 & 100 & 152 & 30 & 82 & 2. & 2.3 & 7.3 & 8.2 & 3.4 & 1.34 & 1.00 & 6.4 & 4.8 & 0.62 \\
\hline 14 & $9 \mathrm{a}$ & 11 & n.d. & 42 & 360 & 267 & 51 & 98 & 102 & 107 & 108 & 37 & 86 & 2. & 2.4 & 8.3 & 8.4 & 3.2 & 1.18 & 0.92 & 6.1 & 3.9 & 0.61 \\
\hline 15 & $9 a$ & 13 & 260 & 45 & 380 & 290 & 32 & 85 & 122 & 102 & 129 & 30 & 100 & 1. & 2.1 & 5.0 & 7. & 2.9 & 1.03 & 0.68 & 4.9 & 3.2 & 0.37 \\
\hline 16 & $9 b$ & 9 & n.d. & 38 & 320 & 264 & 43 & 114 & 98 & 86 & 106 & 30 & 81 & 2.2 & 1.9 & 7.8 & 7.3 & 2.8 & 1.09 & 0.72 & 5.5 & 2.8 & 0.49 \\
\hline 17 & $9 b$ & 11 & 220 & 44 & 360 & 281 & 51 & 97 & 107 & 95 & 122 & 31 & 89 & 2.0 & 1. & 8.8 & 7.0 & 3.0 & 1.27 & 0.85 & 5.4 & 3.0 & 0.53 \\
\hline 18 & $9 c$ & 11 & n.d. & 45 & 340 & 322 & 49 & 115 & 115 & 94 & 129 & 37 & 86 & 2.2 & 2.1 & 8.0 & 5.9 & 3.0 & 1.16 & 0.80 & 5.9 & 3.3 & 0.56 \\
\hline 19 & $9 d$ & 7 & 140 & 38 & 340 & 225 & 42 & 85 & 90 & 92 & 170 & 36 & 74 & 2.9 & 2.1 & 6.1 & 6.9 & 3.0 & 1.11 & 0.77 & 6.1 & 3 & 0.54 \\
\hline 20 & $9 d$ & 7 & n.d. & 39 & 300 & 217 & 41 & 80 & 90 & 90 & 122 & 36 & 88 & 1.5 & 1.8 & 7.2 & 8.1 & 3.1 & 1.38 & 0.80 & 5.2 & 3.3 & 0.47 \\
\hline 21 & $10 \mathrm{a}$ & 7 & 200 & 45 & 420 & 239 & 47 & 78 & 103 & 105 & 105 & 36 & 94 & 2.5 & 2.6 & 8.9 & 6.2 & 3.6 & 1.23 & 0.92 & 6.8 & 4.0 & 0.57 \\
\hline 22 & $10 \mathrm{a}$ & 6 & n.d. & 39 & 340 & 212 & 43 & 76 & 85 & 92 & 131 & 33 & 84 & 2.4 & 2.0 & 9.7 & 9.8 & 3.1 & 1.23 & 0.77 & 4.6 & 3.4 & 0.55 \\
\hline 23 & $10 \mathrm{~b}$ & 7 & 180 & 40 & 340 & 222 & 45 & 100 & 97 & 100 & 118 & 33 & 89 & 2.2 & 2.3 & 9.1 & 8.3 & 3.2 & 1.27 & 0.78 & 4.7 & 3.5 & 0.54 \\
\hline 24 & 11 & 6 & n.d. & 40 & 360 & 221 & 43 & 89 & 90 & 88 & 129 & 30 & 80 & 2.0 & 1.5 & 7.3 & 6.6 & 3.0 & 0.97 & 0.73 & 4.8 & 3.3 & 0.53 \\
\hline 25 & 12 & 7 & 180 & 39 & 340 & 263 & 45 & 105 & 74 & 84 & 122 & 30 & 73 & 2.7 & 2.2 & 6.4 & 6.2 & 2.8 & 1.05 & 0.72 & 5.2 & 3.1 & 0.48 \\
\hline 26 & 12 & 6 & n.d. & 39 & 320 & 262 & 43 & 109 & 78 & 86 & 119 & 37 & 84 & 1.6 & 2.1 & 6.6 & 8.5 & 3.0 & 1.24 & 0.70 & 5.8 & 3.5 & 0.48 \\
\hline 27 & 13 & 7 & 190 & 36 & 280 & 200 & 40 & 80 & 96 & 100 & 112 & 32 & 81 & 1.9 & 1.3 & 6.1 & 6.2 & 2.9 & 0.92 & 0.65 & 5.9 & 3.4 & 0.44 \\
\hline 28 & 13 & 6 & n.d. & 43 & 300 & 255 & 42 & 71 & 92 & 95 & 117 & 31 & 87 & 2.6 & 2.6 & 8.0 & 6.4 & 3.1 & 1.25 & 0.72 & 5.2 & 3.5 & 0.48 \\
\hline 29 & 13 & 7 & 130 & 33 & 240 & 177 & 40 & 100 & 90 & 93 & 116 & 27 & 80 & 1.3 & 2.0 & 6.8 & 5.7 & 2.5 & 0.92 & 0.72 & 5.0 & 2.7 & 0.45 \\
\hline
\end{tabular}

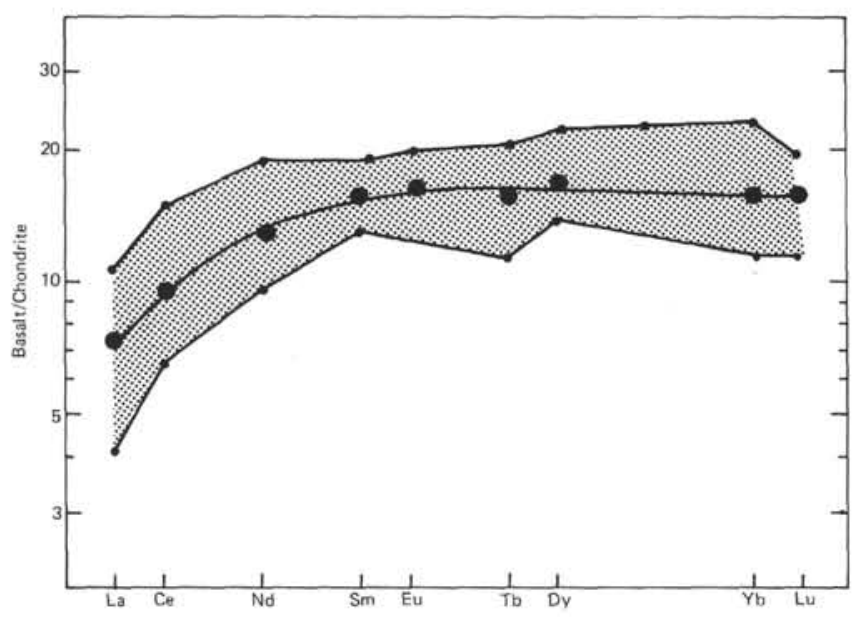

Figure 4. Chondrite-normalized REE distribution patterns of Hole 417D basalts.

$418 \mathrm{~A}$ basalts). On the average, Hole $418 \mathrm{~A}$ basalts are slightly higher in $\mathrm{MgO}$ and $\mathrm{CaO}$, and lower in $\mathrm{TiO}_{2}$, total iron, $\mathrm{Na}_{2} \mathrm{O}$, and $\mathrm{P}_{2} \mathrm{O}_{5}$. But in all cases, the differences are within the range covered by the standard deviation. The overall similarity between the basalts recovered from both holes is especially well displayed by the oxide frequency distributions plotted in Figure 3.

No significant differences in trace-element concentrations have been observed either. All data obtained are summarized in Table 9; means are given in Table 6. The REE patterns exhibit the same strong depletion in LREE as Hole 417D basalts, the average $\mathrm{La} / \mathrm{Sm}$ e.f. being 0.45 in this case. Overall REE enrichment is slightly less than in Hole
TABLE 6

Average Trace Element Contents of Hole 417D and 418A Basalts

\begin{tabular}{|c|c|c|c|c|c|c|c|c|}
\hline \multirow[b]{3}{*}{ Elements } & \multicolumn{2}{|c|}{ 417D Basalts } & \multicolumn{6}{|c|}{ 418A Basalts } \\
\hline & \multirow[b]{2}{*}{$\bar{x}$} & \multirow[b]{2}{*}{$\pm \delta$} & \multicolumn{2}{|c|}{ All Samples } & \multicolumn{2}{|c|}{ Unit $6 b$} & \multicolumn{2}{|c|}{ Unit 13} \\
\hline & & & $\bar{x}$ & $\pm \delta$ & $\bar{x}$ & $\pm \delta$ & $\bar{x}$ & $\pm \delta$ \\
\hline $\mathrm{Li}$ & 9.3 & 2.6 & 8.6 & 2.7 & 7 & 2 & 8 & 1 \\
\hline $\mathrm{F}$ & 220 & 58 & 173 & 45 & 179 & 43 & 167 & 18 \\
\hline $\mathrm{Sc}$ & 43 & 3 & 39 & 2 & 38 & 2 & 38 & 3 \\
\hline V & 346 & 36 & 310 & 35 & 285 & 26 & 326 & 27 \\
\hline $\mathrm{Cr}$ & 252 & 31 & 260 & 52 & 322 & 17 & 220 & 30 \\
\hline Co & 46 & 7 & 44 & 3 & 44 & 3 & 43 & 4 \\
\hline $\mathrm{Ni}$ & 95 & 15 & 101 & 26 & 137 & 12 & 89 & 7 \\
\hline $\mathrm{Cu}$ & 100 & 12 & 109 & 7 & 112 & 4 & 111 & 7 \\
\hline $\mathrm{Zn}$ & 101 & 9 & 84 & 8 & 80 & 6 & 84 & 5 \\
\hline $\mathrm{Sr}$ & 123 & 17 & 114 & 22 & 120 & 23 & 107 & 10 \\
\hline $\mathrm{Y}$ & 32 & 5 & 29 & 5 & 29 & 5 & 29 & 2 \\
\hline $\mathrm{Zr}$ & 86 & 8 & 65 & 12 & 57 & 8 & 60 & 6 \\
\hline Hf & 2.3 & 0.5 & 2.1 & 0.5 & 1.6 & 0.3 & 2.3 & 0.6 \\
\hline
\end{tabular}

417D basalts, which corresponds to the lower averages in $\mathrm{TiO}_{2}$ and $\mathrm{P}_{2} \mathrm{O}_{5}$ (Dietrich et al., 1977).

As within Hole 417D, downhole variation is rather limited. From all stratigraphic units established by the shipboard scientific party, only Units $6 \mathrm{~b}$ and 13 , both representing thick pillow sequences, can be distinguished by chemical criteria. But even in this case, significant differences exist only for $\mathrm{TiO}_{2}, \mathrm{Ni}$, and $\mathrm{Cr}$, while the concentration ranges of all other elements determined largely overlap (see Table 6). In a $\mathrm{Ni}_{-} \mathrm{TiO}_{2}$ plot (Figure 5), all samples from Unit 13 are closely grouped within a small area in the bulk of the data, while Unit $6 \mathrm{~b}$ basalts are clearly separated from all other units by their high $\mathrm{Ni}$ concentrations at low $\mathrm{TiO}_{2}$. Similar observations can be made for chromium, which is also enriched in basalts from Unit $6 \mathrm{~b}$. Minor differences in the average REE enrichment level of the two units are 
TABLE 7

Major Oxide Compositions of Hole 418A Basalts ("raw data" set)

\begin{tabular}{|c|c|c|c|c|c|c|c|c|c|c|c|c|c|c|c|c|c|}
\hline No. & $\begin{array}{c}\text { Sample } \\
\text { (Interval in } \mathrm{cm} \text { ) }\end{array}$ & $\begin{array}{l}\text { Depth } \\
(\mathrm{m})\end{array}$ & Unit & $\mathrm{SiO}_{2}$ & $\mathrm{TiO}_{2}$ & $\mathrm{Al}_{2} \mathrm{O}_{3}$ & $\mathrm{Fe}_{2} \mathrm{O}_{3}$ & $\mathrm{FeO}$ & $\mathrm{MgO}$ & $\mathrm{MnO}$ & $\mathrm{CaO}$ & $\mathrm{Na}_{2} \mathrm{O}$ & $\mathrm{K}_{2} \mathrm{O}$ & $\mathrm{P}_{2} \mathrm{O}_{5}$ & $\mathrm{H}_{2} \mathrm{O}^{+}$ & $\mathrm{CO}_{2}$ & Total \\
\hline 1 & $15-2,51-53$ & 322.0 & 1 & 49.0 & 1.22 & 16.5 & 5.04 & 3.96 & 7.73 & 0.12 & 12.0 & 2.50 & 0.08 & 0.11 & 1.75 & 0.1 & 100.01 \\
\hline 2 & $17-1,101-103$ & 333.5 & $2 \mathrm{~b}$ & 48.8 & 1.21 & 17.6 & 4.88 & 3.01 & 8.21 & 0.10 & 11.1 & 2.83 & 0.11 & 0.17 & 1.88 & 0.33 & 100.23 \\
\hline 3 & $18-1,38-40$ & 339.4 & $2 \mathrm{c}$ & 48.5 & 1.07 & 16.3 & 3.96 & 5.57 & 7.46 & 0.15 & 13.1 & 2.24 & 0.06 & 0.12 & 1.44 & 0.34 & 100.31 \\
\hline 4 & $18-3,121-123$ & 343.2 & $2 \mathrm{c}$ & 49.6 & 1.04 & 15.4 & 3.81 & 5.84 & 8.12 & 0.15 & 12.3 & 2.32 & 0.08 & 0.19 & 1.22 & 0.11 & 100.18 \\
\hline 5 & $19-6,5-7$ & 355.6 & $2 \mathrm{c}$ & 49.6 & 1.19 & 15.5 & 3.71 & 6.32 & 7.71 & 0.16 & 12.4 & 2.25 & 0.07 & 0.11 & 1.05 & 0.10 & 100.17 \\
\hline 6 & $20-2,20-22$ & 358.7 & $2 \mathrm{c}$ & 49.1 & 1.10 & 15.9 & 4.15 & 5.09 & 7.29 & 0.16 & 12.9 & 2.30 & 0.07 & 0.11 & 1.21 & 0.77 & 100.15 \\
\hline 7 & $24-1,95-97$ & 377.0 & 3 & 48.2 & 1.22 & 17.0 & 5.61 & 3.70 & 7.05 & 0.11 & 12.0 & 2.38 & 0.15 & 0.10 & 1.93 & 0.45 & 99.90 \\
\hline 8 & $26-1,91-93$ & 385.4 & 4 & 48.4 & 1.15 & 16.5 & 4.41 & 4.85 & 6.96 & 0.15 & 12.9 & 2.30 & 0.05 & 0.10 & 1.63 & 0.93 & 100.33 \\
\hline 9 & $27-1,36-38$ & 393.9 & 5 & 46.3 & 1.21 & 15.1 & 5.36 & 4.70 & 7.21 & 0.21 & 13.4 & 2.27 & 0.20 & 0.11 & & 2.47 & 100.54 \\
\hline 10 & $28-5,31-33$ & 408.8 & 5 & 47.7 & 1.26 & 17.0 & 4.71 & 4.45 & 7.29 & 0.20 & 12.2 & 2.39 & 0.13 & 0.11 & 1.53 & 1.03 & 100.00 \\
\hline 11 & $30-1,63-65$ & 415.1 & 5 & 47.4 & 1.20 & 18.4 & 5.01 & 3.66 & 6.63 & 0.17 & 12.7 & 2.37 & 0.18 & 0.10 & & 0.86 & 100.26 \\
\hline 12 & $33-6,7-9$ & 447.6 & 5 & 48.6 & 1.20 & 18.3 & 5.53 & 3.97 & 6.47 & 0.14 & 10.8 & 2.45 & 0.44 & 0.11 & 1.61 & 0.51 & 100.13 \\
\hline 13 & $36-4,10-12$ & 471.6 & 5 & 49.3 & 1.23 & 16.9 & 3.86 & 4.70 & 6.22 & 0.20 & 13.4 & 2.33 & 0.03 & 0.12 & & 0.32 & 99.97 \\
\hline 14 & $38-5,119-121$ & 486.7 & 5 & 49.5 & 1.38 & 17.0 & 4.90 & 3.19 & 7.38 & 0.18 & 11.6 & 2.55 & 0.10 & 0.13 & 1.59 & 0.60 & 100.10 \\
\hline 15 & $40-1,105-107$ & 498.6 & 5 & 46.9 & 1.20 & 17.0 & 5.10 & 3.78 & 6.13 & 0.17 & 12.7 & 2.24 & 0.98 & 0.16 & & 1.30 & 100.09 \\
\hline 16 & $41-4,32-34$ & 503.3 & $6 a$ & 46.7 & 1.23 & 18.3 & 5.51 & 4.68 & 5.80 & 0.20 & 12.9 & 2.36 & 0.25 & 0.12 & 1.82 & 0.45 & 100.32 \\
\hline 17 & $43-3,66-68$ & 520.2 & $6 b$ & 47.9 & 1.00 & 17.2 & 3.62 & 4.88 & 6.88 & 0.16 & 13.6 & 2.12 & 0.03 & 0.11 & & 0.94 & 99.94 \\
\hline 18 & $44-3,54-56$ & 529.1 & $6 \mathrm{~b}$ & 48.3 & 1.05 & 16.6 & 4.2 & 4.87 & 6.30 & 0.14 & 13.4 & 2.3 & 0.03 & 0.09 & 2.11 & 0.69 & 100.08 \\
\hline 19 & $45-3,118-120$ & 538.7 & $6 \mathrm{~b}$ & 49.0 & 1.16 & 15.3 & 3.5 & 6.39 & 8.04 & 0.17 & 12.2 & 2.30 & 0.06 & 1 & & 0.26 & 100.03 \\
\hline 20 & $45-5,86-88$ & 541.4 & $6 b$ & 47.6 & 0.96 & 17.2 & 4.9 & 4.05 & 5.80 & 0.13 & 14.0 & 2.1 & 0.19 & 0.10 & 1. & 0.92 & 99.79 \\
\hline 21 & $46-5,62-64$ & 550.1 & $6 \mathrm{~b}$ & 46.4 & 0.98 & 15.5 & 3.77 & 5.10 & 6.22 & 0.16 & 15.0 & 2.0 & 0.09 & 0.13 & & 2.70 & 99.88 \\
\hline 22 & $47-2,117-119$ & 555.2 & $6 \mathrm{~b}$ & 48.9 & 1.02 & 16.4 & 4.45 & 5.27 & 7.38 & 0.15 & 12.5 & 2.1 & 0.06 & 0.11 & 1. & 0.34 & 100.28 \\
\hline 23 & $48-2,132-134$ & 564.3 & $6 \mathrm{~b}$ & 46.4 & 1.04 & 15.5 & 4.1 & 4.54 & 5.97 & 0.17 & 15.4 & 2.1 & 0.14 & 0.10 & 1. & 2.67 & 100.13 \\
\hline 24 & $48-5,21-23$ & 567.7 & $6 \mathrm{~b}$ & 47.6 & 1.07 & 17.0 & 5.4 & 3.81 & 5.14 & 0.16 & 14.1 & 2.1 & 0.11 & 010 & 28 & 1.35 & 100.14 \\
\hline 25 & $49-1,126-128$ & 571.3 & $6 \mathrm{~b}$ & 48.5 & 1.12 & 16.7 & 4.6 & 5.04 & 6.22 & 0.17 & 13.0 & 2.2 & 0.04 & 0. & 1.5 & 0.44 & 99.78 \\
\hline 26 & $49-3, \mathrm{CC}$ & 573.6 & $6 \mathrm{~b}$ & 49.1 & 1.07 & 16.9 & 4.2 & 5.28 & 6.80 & 0.18 & 12.5 & 2.0 & 0.03 & 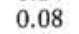 & 1. & 0.30 & 100.22 \\
\hline 27 & $50-2,29-3$ & 575.3 & $6 \mathrm{~b}$ & 50.0 & 1.18 & 15.6 & 4.1 & 5.64 & 7.21 & 0.21 & 12.7 & 2.2 & 0.04 & 0. & 1. & 0.23 & 100.36 \\
\hline 28 & $50-3,32-34$ & 576.8 & $6 \mathrm{~b}$ & 47.9 & 1.06 & 16.9 & 4.70 & 4.93 & 5.97 & 0.18 & 13.4 & 2.0 & 0.13 & $0 .($ & 1. & 0.99 & 100.15 \\
\hline 29 & $52-1,98-100$ & 588.1 & $6 \mathrm{~b}$ & 48.6 & 1.09 & 16.9 & 5.4 & 4.47 & 5.64 & 0.16 & 13.1 & 2. & 0.04 & 0.0 & 1.77 & 0.58 & 99.96 \\
\hline 30 & $51-4,69-71$ & 593.0 & $6 \mathrm{~b}$ & 49.4 & 1.17 & 17.4 & 5.0 & 4.34 & 5.22 & 0.18 & 13. & 2.3 & 0. & 0. & 1.45 & $<0.1$ & 100.17 \\
\hline 31 & $53-3,104-106$ & 605.7 & $6 \mathrm{~b}$ & 47.9 & 1.05 & 16.2 & 3.6 & 5.26 & 6.96 & 0.18 & 13.6 & 2.1 & 0.05 & 0.1 & 1. & 1.60 & 100.05 \\
\hline 32 & $54-2,3$ & 612.8 & 7 & 49.2 & 1.38 & 15.3 & 3.8 & 6.93 & 7.71 & 0.19 & 11 & 2.1 & 0.04 & 0. & 1 & 0.20 & 100.29 \\
\hline 33 & $55-3,1$ & 623.5 & 7 & 48.4 & 1.37 & 15.0 & 4.27 & 6.55 & 7.46 & 0.21 & 12. & 2.0 & 0.06 & 0. & & 0.47 & 99.94 \\
\hline 34 & $56-6,1$ & 638.7 & $8 \mathrm{~b}$ & 48.3 & 1.39 & 16.3 & 4.56 & 5.55 & 6.72 & 0.19 & 12.8 & 2.3 & 0.05 & 0. & & 0.71 & 100.18 \\
\hline 35 & $58-2$ & 650.5 & $8 c$ & 47.9 & 1.51 & 15.4 & 4.25 & 6.15 & 6.80 & 0.22 & 12.8 & 2.33 & 0. & & & 1.62 & 41 \\
\hline 36 & $59-2,1$ & 660.2 & $8 c$ & 47.9 & 1.56 & 16.0 & 5.47 & 5.28 & 5.80 & 0.19 & 12.6 & 2.3 & 0.05 & 0 & & 0.63 & .06 \\
\hline 37 & $60-1,1$ & 667.2 & $8 \mathrm{c}$ & 48.6 & 1.4 & 15.6 & 5.3 & 5.77 & 6.88 & 0.22 & 11.9 & 2.4 & 0.05 & 0 & & 0.46 & 37 \\
\hline 38 & $60-6,33-35$ & 674.8 & 9 & 48.7 & 1.50 & 15.9 & 4.18 & 6.86 & 6.55 & 0.19 & 11.8 & 2.3 & 0.07 & 3 & 1. & 0.28 & 100.25 \\
\hline 39 & $9-111$ & 694.1 & 12 & 48.9 & 1.3 & 16.9 & 4.27 & 5.77 & 6.63 & 0.18 & 12.4 & 2.3 & 0. & & & 0.16 & 100.04 \\
\hline 40 & 64-2, & 3 & 13 & 48.7 & 1.33 & 16.9 & 4.8 & 5.15 & 6.63 & 0.17 & 12.8 & 2.2 & 0. & 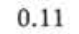 & 0. & 0.21 & 100.10 \\
\hline 41 & 6 & 7 & 13 & 47.8 & 1.3 & 15.6 & 4.1 & 5.79 & 7.63 & 0.22 & 12.7 & 2.2 & 0. & & & 0.76 & .02 \\
\hline 42 & 6 & 712.6 & 13 & 48.1 & 1.1 & 18.6 & 4.2 & 4.36 & 5.64 & 0.17 & 13.4 & 2.1 & 0. & 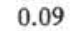 & 1. & 0.86 & 100.17 \\
\hline 43 & 6 & 722.1 & 13 & 48.3 & 1.2 & 15. & 4.4 & 5.99 & 7.3 & 0.21 & 13.3 & 2. & 0. & & & 0.96 & 100.49 \\
\hline 44 & $67-3,6$ & 727.7 & 13 & 48. & 1.2 & 16. & 3.5 & 6.36 & 7.13 & 0.18 & 12.5 & 2.2 & 0. & 0.1 & 1. & 0.40 & 99.82 \\
\hline 45 & & 732 & 13 & 47 & 1.2 & 15.9 & 3.9 & 5.91 & 7.30 & 0.20 & 13.4 & 2.1 & & & & 1.09 & 99.88 \\
\hline 46 & 7 & 748.7 & 13 & 47. & 1.35 & 15. & 3.5 & 5.66 & 6.96 & 0.20 & 13.3 & 2.2 & 0. & 0.1 & 1. & 2.00 & .53 \\
\hline 47 & & 7 & 13 & 48.3 & 1.41 & 14.9 & 4.2 & 6.46 & 8.04 & 0.21 & 12.5 & 2.3 & 0. & & & 0.64 & 100.19 \\
\hline 48 & & 7 & 13 & 49 & 1.3 & 15.1 & 49 & 5.59 & 7.38 & 0.20 & 12.3 & 2.2 & 0. & & 1. & 0.75 & 100.20 \\
\hline 49 & & 756 & 13 & 47.7 & 1.3 & 16. & 4.0 & 5.07 & 6.72 & 0.19 & 13.9 & 2.2 & & & & & 100.53 \\
\hline 50 & & 777. & 13 & 49.4 & 1.2 & 15. & 38 & 6.27 & 7.30 & 0.18 & 12.1 & 2.5 & 0. & 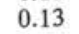 & 1. & 0.09 & 99.84 \\
\hline 51 & & 7 & 13 & 47. & 1.21 & 16. & 4.0 & 5.52 & 7.30 & 0.19 & 13.7 & 2. & 0. & & & & 100.25 \\
\hline 52 & & 788. & $14 a$ & 48. & 1.2 & 16 & 4.6 & 4.78 & 7.54 & 0.1 & 12.3 & 2.3 & 0. & & 1. & 0.75 & 100.13 \\
\hline 53 & & 809 & $14 \mathrm{~b}$ & 48.7 & 1.61 & 14.9 & 4.1 & 6.71 & 6.47 & 0.2 & 12.1 & 2.7 & 0.09 & & & & 99.92 \\
\hline 54 & & 8 & $14 \mathrm{~b}$ & 49.7 & 1.3 & 16 & & & 7.21 & 0.1 & 12. & 2.2 & 0.07 & 0 & 0. & 0.42 & 100.08 \\
\hline 55 & & & $14 \mathrm{~b}$ & 49.7 & 1.2 & 16 & 3.9 & 6.0 & 6.47 & 0.18 & 12.6 & 2.2 & 0.07 & 0. & & 0.49 & 100.28 \\
\hline 56 & 2 & & $15 \mathrm{~b}$ & 49.0 & 1.1 & 16 & 31 & & 6.8 & 0.16 & 12.8 & 2.2 & 0.04 & & 1. & 1.05 & 100.25 \\
\hline 57 & & 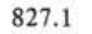 & $14 c$ & 48.7 & 1.2 & 16 & 3.8 & 5.96 & 6.63 & 0.17 & 12.7 & 2.20 & 0.04 & 0.10 & 1.30 & 0.70 & 100.03 \\
\hline 58 & & & $14 \mathrm{c}$ & 49. & 1.4 & & & & 7.3 & 0.1 & 11.8 & 2.2 & & & 0.91 & $<0.1$ & 100.36 \\
\hline 59 & 150 & 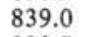 & $14 \mathrm{c}$ & 48.8 & 1.3 & 14 & & & 7.79 & 0.1 & 12 & 2. & 0. & & & 0.31 & 100.06 \\
\hline 60 & & & $14 c$ & & 1.3 & 15 & & & 7.38 & 0.1 & 12. & 2.2 & & & 1. & 0.70 & 100.11 \\
\hline 61 & 47 & & $14 \mathrm{c}$ & 49.2 & 1.1 & 16 & 3.3 & & 7.2 & 0. & 12 & 2. & & 0. & & 0.73 & 100.04 \\
\hline $6 ?$ & & & $14 c$ & 48 & & & & & 7.5 & 0.1 & 13.0 & 2.2 & 0.05 & 0. & 1.22 & 0.81 & 00.21 \\
\hline 63 & & & $14 c$ & 48 & 1. & 14.9 & & 6. & 9.29 & 0.1 & 12.0 & 2. & & & 1. & 0.32 & 100.14 \\
\hline 04 & & & 14 & 48 & & 14 & 3.6 & 6.79 & 10.20 & 0.1 & 11.6 & 1.5 & 0. & 0.13 & & 0.21 & 100.36 \\
\hline 65 & 50 & & $14 c$ & 48. & 1. & 14 & & & 7.9 & 0. & & 2. & & 0. & & 0.58 & 100.29 \\
\hline 66 & 0 & & $14 \mathrm{c}$ & 49 & & & 3. & 6. & 8.1 & 0.1 & 12 & 2.0 & 0. & 0.10 & 1.22 & 0.81 & 100.33 \\
\hline 67 & $6-4,67-69$ & 865.2 & 16 & 48.8 & 1.30 & 14.6 & 4.33 & 6.14 & 7.79 & 0.24 & 12.4 & 2.09 & 0.05 & 0.12 & 1.92 & 0.49 & 100.27 \\
\hline
\end{tabular}

shown in Figure 6. The slightly higher REE concentrations of basalts from Unit 13 correlate with their higher $\mathrm{TiO}_{2}$ contents.

\section{DISCUSSION}

The following facts can be derived from the chemical results:
1) The basalts selected from Holes 417D and 418A exhibit only a rather limited range of chemical variation and are very similar in their major and trace element composition.

2) Since they are only slightly affected by secondary processes, the values obtained for the trace elements and the 
TABLE 8

Recalculated Major Oxide Compositions of Hole 418A Basalts

\begin{tabular}{|c|c|c|c|c|c|c|c|c|c|c|c|c|}
\hline No. & $\mathrm{SiO}_{2}$ & $\mathrm{TiO}_{2}$ & $\mathrm{Al}_{2} \mathrm{O}_{3}$ & $\mathrm{Fe}_{2} \mathrm{O}_{3}$ & $\mathrm{FeO}$ & $\mathrm{MgO}$ & $\mathrm{MnO}$ & $\mathrm{CaO}$ & $\mathrm{Na}_{2} \mathrm{O}$ & $\mathrm{K}_{2} \mathrm{O}$ & $\mathrm{P}_{2} \mathrm{O}_{5}$ & $\mathrm{Mg} / \mathrm{Mg}+\mathrm{Fe}^{2+}$ \\
\hline 1 & 50.0 & 1.25 & 16.8 & 1.45 & 7.40 & 7.89 & 0.12 & 12.3 & 2.55 & 0.08 & 0.11 & 0.66 \\
\hline 2 & 50.2 & 1.24 & 18.1 & 1.28 & 6.49 & 8.44 & 0.10 & 11.0 & 2.91 & 0.11 & 0.17 & 0.70 \\
\hline 3 & 49.5 & 1.09 & 16.6 & 1.56 & 7.96 & 7.62 & 0.15 & 13.0 & 2.29 & 0.06 & 0.12 & 0.63 \\
\hline 4 & 50.3 & 1.06 & 15.6 & 1.56 & 8.04 & 8.24 & 0.15 & 12.4 & 2.35 & 0.08 & 0.19 & 0.65 \\
\hline 5 & 50.2 & 1.20 & 15.7 & 1.64 & 8.35 & 7.81 & 0.16 & 12.5 & 2.28 & 0.07 & 0.11 & 0.62 \\
\hline 6 & 50.7 & 1.13 & 16.4 & 1.52 & 8.75 & 7.52 & 0.17 & 12.3 & 2.37 & 0.07 & 0.11 & 0.60 \\
\hline 7 & 49.8 & 1.26 & 17.6 & 1.51 & 7.74 & 7.29 & 0.11 & 11.8 & 2.67 & 0.16 & 0.10 & 0.63 \\
\hline 8 & 50.3 & 1.19 & 17.1 & 1.53 & 7.80 & 7.23 & 0.16 & 12.1 & 2.39 & 0.05 & 0.10 & 0.62 \\
\hline 9 & 50.0 & 1.31 & 16.3 & 1.72 & 8.76 & 7.78 & 0.23 & 11.1 & 2.45 & 0.22 & 0.12 & 0.61 \\
\hline 10 & 49.8 & 1.31 & 17.7 & 1.51 & 7.73 & 7.61 & 0.21 & 11.4 & 2.49 & 0.14 & 0.11 & 0.64 \\
\hline 11 & 49.2 & 1.24 & 19.1 & 1.42 & 7.23 & 6.88 & 0.18 & 12.0 & 2.46 & 0.19 & 0.10 & 0.63 \\
\hline 12 & 50.1 & 1.24 & 18.9 & 1.53 & 7.86 & 6.67 & 0.14 & 10.5 & 2.52 & 0.45 & 0.11 & 0.60 \\
\hline 13 & 50.5 & 1.26 & 17.3 & 1.41 & 7.14 & 6.37 & 0.20 & 13.3 & 2.39 & 0.03 & 0.12 & 0.61 \\
\hline 14 & 51.1 & 1.42 & 17.5 & 1.32 & 6.69 & 7.62 & 0.19 & 11.3 & 2.63 & 0.10 & 0.13 & 0.67 \\
\hline 15 & 49.7 & 1.27 & 18.0 & 1.49 & 7.56 & 6.49 & 0.18 & 11.8 & 2.37 & 1.04 & 0.17 & 0.60 \\
\hline 16 & 48.1 & 1.27 & 18.8 & 1.65 & 8.46 & 5.97 & 0.21 & 12.7 & 2.43 & 0.26 & 0.12 & 0.56 \\
\hline 17 & 49.8 & 1.03 & 17.9 & 1.44 & 7.36 & 7.15 & 0.17 & 12.9 & 2.20 & 0.03 & 0.11 & 0.63 \\
\hline 18 & 50.2 & 1.09 & 17.3 & 1.50 & 7.68 & 6.55 & 0.15 & 13.0 & 2.39 & 0.03 & 0.09 & 0.60 \\
\hline 19 & 50.1 & 1.19 & 15.6 & 1.64 & 8.35 & 8.22 & 0.17 & 12.2 & 2.35 & 0.06 & 0.11 & 0.64 \\
\hline 20 & 49.6 & 1.00 & 17.9 & 1.53 & 7.81 & 6.04 & 0.14 & 13.4 & 2.19 & 0.20 & 0.10 & 0.58 \\
\hline 21 & 50.5 & 1.07 & 16.9 & 1.56 & 7.98 & 6.77 & 0.17 & 12.6 & 2.20 & 0.10 & 0.14 & 0.60 \\
\hline 22 & 50.0 & 1.04 & 16.8 & 1.58 & 8.09 & 7.55 & 0.15 & 12.4 & 2.20 & 0.06 & 0.11 & 0.62 \\
\hline 23 & 50.4 & 1.13 & 16.8 & 1.50 & 7.68 & 6.49 & 0.18 & 13.2 & 2.28 & 0.15 & 0.11 & 0.60 \\
\hline 24 & 50.2 & 1.13 & 17.9 & 1.59 & 8.04 & 5.42 & 0.17 & 13.1 & 2.24 & 0.12 & 0.11 & 0.55 \\
\hline 25 & 50.0 & 1.15 & 17.2 & 1.58 & 8.08 & 6.45 & 0.18 & 12.9 & 2.31 & 0.04 & 0.14 & 0.59 \\
\hline 26 & 50.3 & 1.10 & 17.3 & 1.56 & 7.96 & 6.97 & 0.18 & 12.4 & 2.10 & 0.03 & 0.08 & 0.61 \\
\hline 27 & 50.7 & 1.20 & 15.9 & 1.57 & 8.06 & 7.31 & 0.21 & 12.7 & 2.21 & 0.04 & 0.10 & 0.62 \\
\hline 28 & 50.0 & 1.11 & 17.6 & 1.59 & 8.14 & 6.23 & 0.19 & 12.7 & 2.16 & 0.14 & 0.08 & 0.58 \\
\hline 29 & 50.3 & 1.13 & 17.5 & 1.62 & 8.27 & 5.84 & 0.17 & 12.8 & 2.15 & 0.04 & 0.09 & 0.56 \\
\hline 30 & 50.2 & 1.19 & 17.7 & 1.51 & 7.71 & 5.35 & 0.18 & 13.6 & 2.42 & 0.04 & 0.12 & 0.55 \\
\hline 31 & 50.5 & 1.11 & 17.1 & 1.49 & 7.62 & 7.33 & 0.19 & 12.2 & 2.23 & 0.05 & 0.14 & 0.63 \\
\hline 32 & 50.1 & 1.41 & 15.6 & 1.76 & 8.98 & 7.85 & 0.19 & 11.7 & 2.23 & 0.04 & 0.14 & 0.61 \\
\hline 33 & 49.6 & 1.40 & 15.4 & 1.78 & 9.07 & 7.65 & 0.22 & 12.5 & 2.14 & 0.06 & 0.13 & 0.60 \\
\hline 34 & 49.7 & 1.43 & 16.8 & 1.65 & 8.45 & 6.91 & 0.20 & 12.3 & 2.40 & 0.05 & 0.14 & 0.59 \\
\hline 35 & 50.2 & 1.58 & 16.2 & 1.74 & 8.90 & 7.12 & 0.23 & 11.3 & 2.44 & 0.07 & 0.17 & 0.59 \\
\hline 36 & 49.7 & 1.62 & 16.6 & 1.76 & 9.04 & 6.02 & 0.20 & 12.4 & 2.47 & 0.05 & 0.16 & 0.54 \\
\hline 37 & 49.7 & 1.48 & 16.2 & 1.81 & 9.23 & 7.04 & 0.23 & 11.5 & 2.47 & 0.05 & 0.23 & 0.58 \\
\hline 38 & 49.8 & 1.53 & 16.3 & 1.80 & 9.26 & 6.70 & 0.19 & 11.8 & 2.41 & 0.07 & 0.13 & 0.56 \\
\hline 39 & 49.6 & 1.35 & 17.2 & 1.63 & 8.31 & 6.73 & 0.18 & 12.4 & 2.40 & 0.04 & 0.15 & 0.59 \\
\hline 40 & 49.5 & 1.35 & 17.2 & 1.62 & 8.28 & 6.74 & 0.17 & 12.7 & 2.24 & 0.03 & 0.11 & 0.59 \\
\hline 41 & 49.5 & 1.39 & 16.1 & 1.65 & 8.42 & 7.89 & 0.23 & 12.2 & 2.37 & 0.12 & 0.13 & 0.63 \\
\hline 42 & 49.8 & 1.23 & 19.2 & 1.41 & 7.20 & 5.84 & 0.18 & 12.7 & 2.27 & 0.03 & 0.09 & 0.59 \\
\hline 43 & 49.9 & 1.32 & 15.9 & 1.63 & 8.23 & 7.61 & 0.22 & 12.6 & 2.31 & 0.06 & 0.13 & 0.62 \\
\hline 44 & 50.1 & 1.31 & 16.4 & 1.63 & 8.34 & 7.30 & 0.19 & 12.3 & 2.31 & 0.04 & 0.12 & 0.61 \\
\hline 45 & 49.4 & 1.31 & 16.5 & 1.65 & 8.37 & 7.59 & 0.21 & 12.6 & 2.19 & 0.05 & 0.13 & 0.62 \\
\hline 46 & 50.8 & 1.43 & 16.3 & 1.60 & 8.19 & 7.38 & 0.21 & 11.5 & 2.40 & 0.04 & 0.15 & 0.62 \\
\hline 47 & 49.5 & 1.44 & 15.3 & 1.76 & 8.99 & 8.24 & 0.22 & 12.0 & 2.37 & 0.06 & 0.16 & 0.62 \\
\hline 48 & 50.6 & 1.38 & 15.5 & 1.72 & 8.79 & 7.59 & 0.21 & 11.7 & 2.26 & 0.03 & 0.11 & 0.61 \\
\hline 49 & 49.8 & 1.36 & 17.0 & 1.52 & 7.78 & 7.02 & 0.20 & 12.8 & 2.36 & 0.05 & 0.14 & 0.62 \\
\hline 50 & 50.3 & 1.31 & 15.8 & 1.65 & 8.42 & 7.43 & 0.18 & 12.1 & 2.60 & 0.03 & 0.13 & 0.61 \\
\hline 51 & 49.5 & 1.26 & 16.8 & 1.60 & 8.12 & 7.63 & 0.20 & 12.4 & 2.26 & 0.05 & 0.13 & 0.63 \\
\hline 52 & 49.7 & 1.31 & 17.1 & 1.53 & 7.85 & 7.78 & 0.18 & 11.8 & 2.47 & 0.08 & 0.15 & 0.64 \\
\hline 53 & 50.6 & 1.67 & 15.5 & 1.82 & 9.29 & 6.72 & 0.23 & 11.1 & 2.80 & 0.09 & 0.16 & 0.56 \\
\hline 54 & 50.7 & 1.33 & 16.3 & 1.61 & 8.22 & 7.35 & 0.18 & 11.8 & 2.32 & 0.07 & 0.12 & 0.61 \\
\hline 55 & 50.7 & 1.28 & 16.5 & 1.63 & 8.31 & 6.60 & 0.18 & 12.2 & 2.28 & 0.07 & 0.12 & 0.59 \\
\hline 56 & 50.6 & 1.19 & 17.4 & 1.49 & 7.62 & 7.11 & 0.17 & 12.0 & 2.29 & 0.04 & 0.12 & 0.62 \\
\hline 57 & 50.2 & 1.32 & 16.9 & 1.63 & 8.29 & 6.84 & 0.18 & 12.2 & 2.27 & 0.04 & 0.10 & 0.60 \\
\hline 58 & 50.2 & 1.41 & 16.1 & 1.71 & 8.71 & 7.43 & 0.17 & 11.8 & 2.29 & 0.04 & 0.16 & 0.60 \\
\hline 59 & 49.8 & 1.36 & 15.2 & 1.72 & 8.76 & 7.95 & 0.17 & 12.6 & 2.27 & 0.05 & 0.11 & 0.62 \\
\hline 60 & 50.3 & 1.34 & 16.2 & 1.60 & 8.18 & 7.59 & 0.18 & 12.1 & 2.32 & 0.04 & 0.13 & 0.62 \\
\hline 61 & 50.7 & 1.20 & 16.7 & 1.57 & 8.02 & 7.44 & 0.16 & 11.8 & 2.21 & 0.06 & 0.10 & 0.62 \\
\hline 62 & 50.0 & 1.27 & 16.8 & 1.50 & 7.66 & 7.77 & 0.19 & 12.3 & 2.32 & 0.05 & 0.12 & 0.64 \\
\hline 63 & 50.0 & 1.17 & 15.2 & 1.58 & 8.09 & 9.53 & 0.18 & 12.0 & 2.09 & 0.06 & 0.11 & 0.68 \\
\hline 64 & 49.3 & 1.12 & 15.0 & 1.63 & 8.29 & 10.60 & 0.19 & 11.7 & 1.99 & 0.07 & 0.13 & 0.69 \\
\hline 65 & 50.3 & 1.26 & 15.4 & 1.69 & 8.58 & 8.20 & 0.19 & 12.0 & 2.31 & 0.05 & 0.10 & 0.63 \\
\hline 66 & 50.7 & 1.27 & 15.1 & 1.66 & 8.48 & 8.36 & 0.19 & 11.9 & 2.08 & 0.08 & 0.10 & 0.64 \\
\hline 67 & 50.3 & 1.34 & 15.0 & 1.72 & 8.81 & 8.03 & 0.25 & 12.2 & 2.15 & 0.05 & 0.12 & 0.62 \\
\hline
\end{tabular}


TABLE 9

Trace Element Chemistry of Hole 418A Basalts

\begin{tabular}{|c|c|c|c|c|c|c|c|c|c|c|c|c|c|c|c|c|c|c|c|c|c|c|c|}
\hline No. & Unit & $\mathrm{Li}$ & F & $\mathrm{Sc}$ & $\mathrm{V}$ & $\mathrm{Cr}$ & Co & $\mathrm{Ni}$ & $\mathrm{Cu}$ & $\mathrm{Zn}$ & $\mathrm{Sr}$ & $\mathrm{Y}$ & $\mathrm{Zr}_{\mathrm{r}}$ & $\mathrm{Hf}$ & $\mathrm{La}$ & $\mathrm{Ce}$ & $\mathrm{Nd}$ & $\mathrm{Sm}$ & $\mathrm{Eu}$ & $\mathrm{Tb}$ & Dy & $\mathrm{Yb}$ & $\mathrm{Lu}$ \\
\hline 1 & 1 & 13 & 310 & 40 & 280 & 231 & 42 & 88 & 126 & 98 & 131 & 30 & 76 & 2.3 & 1.6 & 0 & 5.9 & 2.6 & .99 & .72 & 5.3 & 2.7 & 0.38 \\
\hline 2 & $2 b$ & 19 & n.d. & 42 & 280 & 260 & 50 & 92 & 128 & 94 & 147 & & 77 & & 1.8 & & & & & 0.75 & 5.4 & 2.7 & 0.36 \\
\hline 3 & $2 c$ & 11 & 110 & 37 & 220 & 212 & 42 & 82 & 102 & 92 & 126 & 34 & 61 & & 1.9 & & & & & .62 & 5.2 & 2.8 & 0.40 \\
\hline 4 & $2 c$ & 11 & n.d. & 36 & 200 & 233 & 41 & 76 & 106 & 94 & 112 & 30 & 62 & & 1.8 & & & & & .64 & 5.4 & 2.5 & 0.38 \\
\hline 5 & $2 c$ & 9 & 100 & 39 & 300 & 211 & 42 & 76 & 110 & 88 & 117 & 34 & 70 & 2.2 & 2.1 & 5.2 & 7.9 & 2.5 & & & 5.6 & & 0.49 \\
\hline 6 & $2 c$ & 9 & n.d. & 35 & 280 & 216 & 39 & 92 & 106 & 80 & 122 & 27 & 55 & 1.2 & 1.6 & 4.8 & 5.8 & 2.3 & 0.80 & 0.68 & 5.3 & 2.6 & 0.43 \\
\hline 7 & 3 & 9 & 240 & 40 & 300 & 262 & 46 & 82 & 118 & 100 & 140 & 26 & 69 & 2.5 & 2.3 & 5.4 & 7.8 & 2.5 & 1.27 & 0.61 & 5.5 & 2.6 & 0.40 \\
\hline 8 & 4 & 9 & n.d. & 39 & 340 & 229 & 42 & 76 & 106 & 78 & 127 & 30 & 65 & 1.9 & 2.0 & 4.5 & 4.8 & 2.5 & 1.03 & 0.77 & 5.8 & 3.0 & 0.38 \\
\hline 9 & 5 & 9 & 210 & 38 & 340 & 280 & 42 & 98 & 100 & $86^{\circ}$ & 96 & 31 & 63 & 2.2 & 2.1 & 6.2 & 6.3 & 2.6 & 0.86 & 0.59 & 6.2 & 3.4 & 0.46 \\
\hline 10 & 5 & 9 & n.d. & 39 & 280 & 280 & 49 & 102 & 110 & 88 & 116 & 35 & 82 & 1.4 & 2.2 & 7.1 & 8.2 & 2.6 & 1.15 & 0.58 & 6.1 & 3.1 & 0.44 \\
\hline 11 & 5 & 9 & 150 & 37 & 320 & 256 & 47 & 102 & 110 & 88 & 109 & 26 & 69 & 1.8 & 2.0 & 5.0 & 6.1 & 2.3 & 0.93 & 0.59 & 5.0 & 2.9 & 0.46 \\
\hline 12 & 5 & 9 & n.d. & 39 & 340 & 297 & 42 & 72 & 105 & 92 & 122 & 28 & 74 & 2.5 & 2.2 & 4.8 & 5.2 & 2.6 & 1.14 & 0.62 & 5.2 & 3.0 & 0.42 \\
\hline 13 & 5 & 9 & 185 & 38 & 320 & 244 & 41 & 102 & 102 & 80 & 128 & 35 & 71 & 2.8 & 1.6 & & 7.1 & & & & & 2.7 & 0.45 \\
\hline 14 & 5 & 11 & n.d. & 42 & 340 & 267 & 46 & 102 & 118 & 88 & 140 & 34 & 94 & 2.1 & 2.4 & & & & & & & & 0.38 \\
\hline 15 & 5 & 20 & 240 & 37 & 280 & 243 & 43 & 88 & 100 & 94 & 172 & 38 & 78 & 2.9 & 1.9 & 7. & 6. & 2.4 & & 0.61 & 4.1 & & 0.42 \\
\hline 16 & $6 a$ & 11 & n.d. & 39 & 340 & 316 & 44 & 116 & 118 & 90 & 187 & 41 & 72 & 2.0 & 1. & 5. & 5 & 2. & & & 4.1 & 2.7 & 0.48 \\
\hline 17 & $6 \mathrm{~b}$ & 7 & 205 & 38 & 280 & 341 & 44 & 134 & 114 & 76 & 176 & 29 & 46 & 1.4 & 2. & 7 & 5 & 2. & & & 4.0 & 2.8 & 0.40 \\
\hline 18 & $6 b$ & 7 & n.d. & 37 & 220 & 292 & 41 & 135 & 114 & 74 & 109 & 26 & 59 & 1.5 & 1. & 4 & 5 & 2 & & & 3.8 & 2.6 & 0.41 \\
\hline 19 & $6 b$ & 9 & 100 & 41 & 320 & 317 & 47 & 128 & 118 & 82 & 102 & 38 & 66 & 1.6 & 1.3 & 4. & 5 & 2.2 & & & 4.0 & 2.9 & 0.46 \\
\hline 20 & $6 b$ & 7 & n.d. & 36 & 260 & 334 & 47 & 119 & 106 & 74 & 164 & 31 & 56 & 1.1 & 0.9 & 2 & 4 & 2.0 & & & & 2.5 & 0.38 \\
\hline 21 & $6 b$ & 9 & 210 & 35 & 280 & 309 & 38 & 125 & 110 & 74 & 119 & 27 & 57 & 1.5 & 2 & 8 & 10 & & & & & 2.6 & 0.41 \\
\hline 22 & $6 b$ & 9 & n.d. & 37 & 280 & 319 & 40 & 124 & 110 & 76 & 114 & 3 & 6 & 1.4 & 1. & 4 & 5 & 2 & & & & 2.6 & 0.38 \\
\hline 23 & $6 b$ & 9 & 220 & 35 & 280 & 297 & 43 & 128 & 106 & 86 & 124 & 3 & 5 & 2. & 1. & 4 & 5 & 2. & & & & 2.6 & 0.39 \\
\hline 24 & $6 b$ & 6 & n.d. & 36 & 260 & 312 & 40 & 138 & 118 & 8 & 1 & 3 & 5 & 2 & 1 & 4 & 5 & & & & & 5 & 0.45 \\
\hline 25 & $6 b$ & 5 & 170 & 39 & 320 & 333 & 44 & 145 & 112 & 8 & 130 & 3 & 71 & 1.7 & 1. & 5 & 6 & & & & & & 0.40 \\
\hline 26 & $6 b$ & 5 & n.d. & 38 & 300 & 327 & 45 & 147 & 112 & 72 & 94 & 2 & 53 & 1.7 & 1. & 5 & 4 & 2 & & & 3.9 & 2.7 & 0.34 \\
\hline 27 & $6 b$ & 5 & 200 & 40 & 300 & 349 & 46 & 154 & 116 & 80 & 110 & 23 & 52 & 2.0 & 1. & 6. & 7 & 2 & & & 4.7 & 2.8 & 0.50 \\
\hline 28 & $6 b$ & 4 & n.d. & 38 & 280 & 330 & 44 & 129 & 108 & 8 & 112 & 22 & 43 & 1.6 & 2.0 & 5 & 5 & 2.1 & & & 4.1 & 2.5 & 0.39 \\
\hline 29 & $6 b$ & 6 & n.d. & 38 & 290 & 326 & 44 & 142 & 116 & 7 & 98 & 22 & 52 & 1.4 & 1.4 & 5 & 8 & 2.1 & & & 4.6 & 2.5 & 0.44 \\
\hline 30 & $6 \mathrm{~b}$ & 3 & 130 & 38 & 320 & 301 & 45 & 142 & 116 & 7 & 109 & 2 & 54 & 1.4 & 1.5 & 7. & 7.0 & 2.8 & & & 4.3 & 2.4 & 0.41 \\
\hline 31 & $6 b$ & 8 & 200 & 38 & 290 & 342 & 49 & 162 & 112 & 9. & 128 & 3. & 66 & 1.7 & 2.7 & 5 & 9.2 & 2.7 & & & 4.5 & 3.0 & 0.39 \\
\hline 32 & 7 & 8 & n.d. & 41 & 310 & 232 & 46 & 82 & 114 & 8 & 10 & 2 & 6 & 2. & 1. & 4 & 9.9 & 3.4 & 1. & 0 . & 5.2 & 2.9 & 0.54 \\
\hline 33 & 7 & 8 & 170 & 41 & 320 & 243 & 46 & 86 & 10 & 8 & 87 & 2 & 66 & 2. & 2. & & 7 & & & & 5.3 & 2.9 & 0.38 \\
\hline 34 & $8 b$ & 7 & n.d. & 37 & 320 & 22 & 4 & 76 & 99 & 8 & 106 & $\mathrm{n}$. & 6 & 2.1 & 2. & 6 & 8 & & & & 5.6 & 3.2 & 0.49 \\
\hline 35 & $8 c$ & 8 & 230 & 38 & 3. & 21 & 42 & 81 & 110 & 9 & 110 & $n$ & 67 & 2.6 & 2. & 6 & n.d & 3. & & & 6 & 3.2 & 0.49 \\
\hline 36 & $8 c$ & 8 & n.d & 41 & 36 & 23 & 46 & 98 & 105 & & 1 & $\mathrm{n}$ & 76 & 2.6 & 2. & 5 & 9 & 3 & & & & 5 & 0.59 \\
\hline 37 & $8 c$ & 8 & 170 & 40 & $3 t$ & 215 & 47 & 92 & 106 & 9 & 112 & $\mathrm{n}$. & 66 & 2.5 & 2. & 6 & 9 & 3 & 1 & & & & 0.52 \\
\hline 38 & 9 & 11 & n.d. & 39 & 360 & 200 & 43 & 7 & 98 & g & 106 & 3. & 74 & 3.3 & 2.5 & 8. & 8 & 2 & 1. & 0 & 6 & 3.3 & 0.51 \\
\hline 39 & 12 & 7 & 130 & 37 & 330 & 220 & 41 & 87 & 95 & 9 & 104 & 3 & 65 & 1.7 & 2.3 & 4.8 & 10.5 & 3.3 & 1. & & 3 & 2.9 & 0.48 \\
\hline 40 & 13 & 8 & n.d. & 38 & 340 & 207 & 41 & 80 & 98 & 79 & 88 & 27 & 58 & 1.9 & 1.6 & 6.7 & 6.7 & 2.6 & 1.00 & 0.69 & 5.8 & 2.9 & 0.49 \\
\hline 41 & 13 & 8 & 140 & 39 & 350 & 213 & 42 & 82 & 110 & 92 & 104 & 29 & 62 & 1.7 & 2.2 & 6.4 & 8.9 & 3.1 & 1.38 & 0.91 & 6.1 & 3.2 & 0.49 \\
\hline 42 & 13 & 10 & n.d. & 32 & 260 & 185 & 38 & 90 & 122 & 85 & 110 & 3 & 5 & 1.4 & 1.8 & 5 & 6 & 2.2 & 1.00 & 0.59 & 5.0 & 2.4 & 0.36 \\
\hline 43 & 13 & 7 & 150 & 39 & 290 & 253 & 44 & 98 & 112 & & 1 & 3 & 5 & 2 & 1 & 4 & 7 & 3.1 & & & 4.6 & 2.9 & 0.48 \\
\hline 44 & 13 & 8 & n.d. & 39 & 330 & 238 & 44 & 87 & 1 & & 1 & & 6 & & & & & & & & 4.3 & 2.9 & 0.47 \\
\hline 45 & 13 & 8 & 170 & 40 & 330 & 248 & 44 & 8 & 1 & & 1 & & 6 & & 2 & & & & & & 5 & 3.2 & 0.52 \\
\hline 46 & 13 & 7 & n.d. & 41 & 330 & 2 & 46 & 9 & 1 & & 1 & n.d. & 52 & 2. & 2. & & & & & & & 9 & 0.47 \\
\hline 47 & 13 & 9 & 190 & 42 & 34 & 1 & 4 & 9 & 1 & & 105 & 26 & 52 & 2.7 & 2.8 & 12. & 5 & & & & & 2 & 0.49 \\
\hline 48 & 1 & 8 & & 4 & 3. & 2 & 4 & & 116 & 07 & 112 & n.d. & n.d. & 2.7 & 2.0 & 5. & 8.2 & 2.6 & 1.05 & & & 0 & 0.47 \\
\hline 49 & 13 & 8 & 170 & 39 & 35 & 228 & 4 & & 112 & 80 & 116 & n.d. & 62 & 3.1 & 2.5 & 6.4 & 5.5 & 3.2 & 1.22 & & 5.4 & .0 & 0.47 \\
\hline 50 & 13 & 8 & n.d. & 31 & 310 & 156 & 33 & 76 & 106 & 82 & 102 & n.d. & 58 & 1.2 & 1.7 & 4.5 & 6.0 & 2.5 & 1.01 & 0.52 & 5.2 & 2.4 & 0.37 \\
\hline 51 & 13 & 7 & 180 & 39 & 330 & 231 & 43 & 92 & 10 & & 13 & & 72 & & & & & & & & & 9 & 0.42 \\
\hline 52 & $14 \mathrm{a}$ & 12 & n.d. & 41 & 310 & 242 & 47 & 171 & 12 & & 82 & n.d. & 54 & 2.2 & 2.3 & 5.3 & 7.7 & 2.8 & 1.11 & 0.81 & 6.3 & 2.6 & 0.46 \\
\hline 53 & $14 b$ & 6 & 180 & 39 & 36 & 18 & 44 & 66 & 99 & 01 & 139 & 40 & 108 & 3.2 & 2.9 & 8.3 & 10.0 & 4.3 & 1.32 & 1.11 & 5.6 & 3.7 & 0.57 \\
\hline 54 & $14 b$ & 7 & & 43 & 350 & 219 & 43 & 70 & 109 & 87 & 126 & 40 & 67 & 2.3 & 1.8 & 6.4 & 9.8 & 3.3 & 1.25 & 0.84 & 4.8 & 2.9 & 0.45 \\
\hline 55 & $14 b$ & 10 & 160 & 38 & 300 & 187 & 39 & 64 & 98 & 73 & 84 & 23 & 66 & 2.2 & 2.0 & 4.7 & 10.0 & 2.4 & 0.93 & 0.69 & 5.5 & 2.6 & 0.45 \\
\hline 56 & $15 b$ & 7 & 140 & 37 & 290 & 253 & 40 & 92 & 100 & 74 & 99 & n.d. & 57 & 1.1 & 2.0 & 5.7 & 7.8 & 2.8 & 1.03 & 0.70 & 4.7 & 2.8 & 0.40 \\
\hline 57 & $14 c$ & 9 & n.d. & 38 & 260 & 193 & 41 & 59 & 102 & 77 & 119 & 2 & 6 & 1.8 & 2. & 7. & 7 & 3.1 & 0. & 0. & 5.3 & 2.7 & 0.41 \\
\hline 58 & $14 c$ & 7 & n.d. & 41 & 350 & 207 & 42 & 76 & 102 & 89 & 127 & 4 & 8 & 1. & 2 & 6 & 9 & 3. & & & 5.2 & .1 & 0.52 \\
\hline 59 & $14 c$ & 10 & 140 & 40 & 340 & 229 & 44 & 64 & 98 & 77 & 95 & 2 & 59 & 2.3 & 2 & 7 & 8. & 2.5 & 0.92 & 0. & 1 & 8 & 0.49 \\
\hline 60 & $14 \mathrm{c}$ & 9 & n.d. & 38 & 310 & 249 & 42 & 92 & 109 & 7 & 114 & 28 & 86 & 1.3 & 2.0 & 5.1 & 9.2 & 2.8 & 0.99 & 0.72 & 4.8 & 2.7 & 0.55 \\
\hline 61 & $14 \mathrm{c}$ & 12 & 140 & 36 & 300 & 239 & 43 & 96 & 98 & & 106 & 23 & 53 & 2.1 & 2.8 & 7.8 & 8.4 & 2.2 & 0.94 & 0.54 & 4.5 & 2.5 & 0.45 \\
\hline 62 & $14 \mathrm{c}$ & 7 & n.d. & 38 & 330 & 302 & 44 & 120 & 110 & 93 & 114 & 28 & 86 & 2.9 & 1.8 & 4.9 & 5.1 & 2.5 & 1.16 & 0.69 & 5.6 & 3.0 & 0.45 \\
\hline 63 & $14 \mathrm{c}$ & 9 & 120 & 36 & 310 & 336 & 48 & 123 & 112 & 75 & 94 & 30 & 74 & 2.9 & 1.9 & 6.3 & 9.5 & 2.4 & 1.11 & 0.67 & 5.0 & 2.6 & 0.39 \\
\hline 64 & $14 \mathrm{c}$ & 8 & n.d. & 37 & 3 & 420 & 51 & 108 & 120 & 92 & 87 & 31 & 77 & 2.1 & 1.6 & 6.1 & 10.0 & 2.4 & 0.91 & 0.69 & 4.6 & 2.6 & 0.40 \\
\hline 65 & $14 \mathrm{c}$ & 9 & 150 & 39 & 300 & 287 & 47 & 123 & 114 & 69 & 79 & 23 & 56 & 1.6 & 1.8 & 6.8 & 8.1 & 2.2 & 0.99 & 0.55 & 5.0 & 2.4 & 0.37 \\
\hline 66 & $14 \mathrm{c}$ & 9 & n.d. & 40 & 300 & 290 & 46 & 107 & 104 & 73 & 92 & 17 & 51 & 1.8 & 2.0 & 7.2 & 5.2 & 2.3 & 1.01 & 0.53 & 6.4 & 2.6 & 0.42 \\
\hline 67 & 16 & 9 & 180 & 41 & 340 & 292 & 45 & 101 & 112 & 81 & 76 & 24 & 64 & 2.2 & 2.0 & 7.0 & 8.0 & 2.4 & 1.08 & 0.74 & 4.6 & 2.8 & 0.37 \\
\hline
\end{tabular}

recalculated values in the case of the major oxides constitute the primary composition and variability of this material.

3) In their overall chemistry, the Cretaceous basalts of the "Bermuda Triangle" sites correspond closely to ocean floor basalts drilled or dredged from modern mid-ocean ridge environments. This is clearly demonstrated by a comparison of their major and trace element abundances with the average composition of "abyssal oceanic tholeiites" (last column in Table 1) as compiled by Wedepohl (1975).
4) A characteristic feature of the basalts investigated in contrast to Wedepohl's averages is their very low primary $\mathrm{K}_{2} \mathrm{O}$ content and their pronounced depletion in LREE, as well as other LIL elements.

5) In terms of their normative composition (given in Tables 10 and 11), the Leg 51 to 53 basalts can be classified as (low-K) tholeiites. Most of the samples project in the olivine tholeiite field of the normative ol-di-hy diagram and fall close to the di-hy join (Figure 7). Only a few samples 


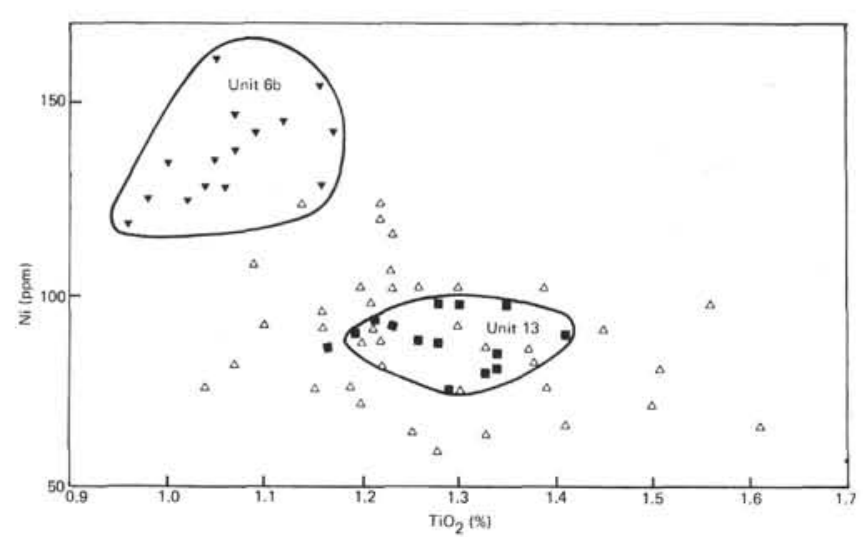

Figure 5. $\mathrm{Ni}-\mathrm{TiO}_{2}$ variation diagram for Hole $418 \mathrm{~A}$ basalts.

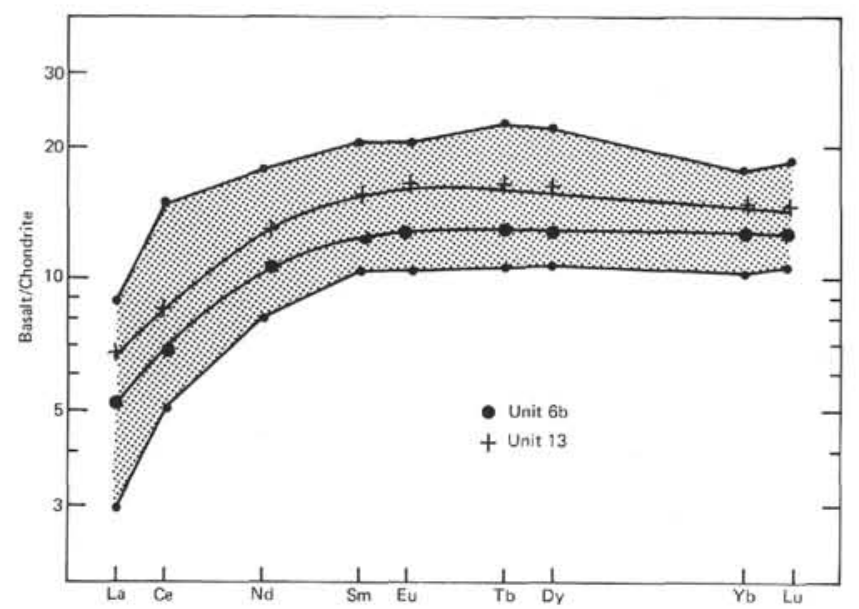

Figure 6. Chondrite-normalized REE distribution patterns of Hole $418 \mathrm{~A}$ basalts.

TABLE 10

CIPW Norms of Hole 417D Basalts

\begin{tabular}{|c|c|c|c|c|c|c|c|c|c|c|c|}
\hline Core & Q & OR & $\mathrm{AB}$ & AN & DI & HY & OL & MT & IL & AP & RAN $^{a}$ \\
\hline 1 & 0.00 & 1.12 & 21.66 & 35.43 & 19.06 & 15.77 & 1.45 & 2.41 & 2.72 & 0.32 & 0.62 \\
\hline 2 & 0.00 & 0.41 & 22.34 & 34.88 & 18.63 & 16.68 & 1.63 & 2.36 & 2.75 & 0.32 & 0.61 \\
\hline 3 & 0.00 & 2.07 & 20.31 & 35.13 & 21.72 & 6.14 & 8.92 & 2.58 & 2.72 & 0.35 & 0.63 \\
\hline 4 & 0.00 & 3.01 & 21.07 & 35.34 & 13.22 & 16.44 & 5.39 & 2.48 & 2.66 & 0.39 & 0.63 \\
\hline 5 & 0.00 & 2.60 & 20.82 & 34.59 & 11.42 . & 23.58 & 0.90 & 2.65 & 2.96 & 0.39 & 0.62 \\
\hline 6 & 0.00 & 0.71 & 21.66 & 32.63 & 20.30 & 14.95 & 3.76 & 2.52 & 3.04 & 0.39 & 0.60 \\
\hline 7 & 0.00 & 0.95 & 22.76 & 34.66 & 14.16 & 18.50 & 2.94 & 2.33 & 3.21 & 0.37 & 0.60 \\
\hline 8 & 0.00 & 0.53 & 25.22 & 36.02 & 11.74 & 20.29 & 0.51 & 2.03 & 3.27 & 0.39 & 0.59 \\
\hline 9 & 2.41 & 0.71 & 23.10 & 31.60 & 19.31 & 17.14 & 0.00 & 2.19 & 3.04 & 0.46 & 0.58 \\
\hline 10 & 0.00 & 0.47 & 22.09 & 33.07 & 19.05 & 18.38 & 1.21 & 2.32 & 2.98 & 0.39 & 0.60 \\
\hline 11 & 0.00 & 0.65 & 20.99 & 34.66 & 18.62 & 13.18 & 5.99 & 2.58 & 2.98 & 0.42 & 0.62 \\
\hline 12 & 0.00 & 0.41 & 20.65 & 33.32 & 19.82 & 13.61 & 5.97 & 2.68 & 3.17 & 0.37 & 0.62 \\
\hline 13 & 0.00 & 0.24 & 20.06 & 30.72 & 23.89 & 13.01 & 5.89 & 2.71 & 3.06 & 0.39 & 0.61 \\
\hline 14 & 0.00 & 1.24 & 20.06 & 34.86 & 17.25 & 15.42 & 5.22 & 2.65 & 3.06 & 0.42 & 0.63 \\
\hline 15 & 0.00 & 1.48 & 23.27 & 37.94 & 10.68 & 17.17 & 4.10 & 2.06 & 2.98 & 0.35 & 0.62 \\
\hline 16 & 0.00 & 0.35 & 21.16 & 39.90 & 16.93 & 9.46 & 7.15 & 2.20 & 2.51 & 0.32 & 0.65 \\
\hline 17 & 0.00 & 0.53 & 22.85 & 37.55 & 15.09 & 14.57 & 4.01 & 2.03 & 3.04 & 0.32 & 0.62 \\
\hline 18 & 0.00 & 0.24 & 23.69 & 36.98 & 18.53 & 6.26 & 9.20 & & 2.72 & & \\
\hline 19 & 0.00 & 0.24 & 20.22 & 38.54 & 15.73 & 16.02 & 3.73 & 2.52 & 2.72 & 0.30 & 0.66 \\
\hline 20 & 0.00 & 0.24 & 21.16 & 40.23 & 13.09 & 19.41 & 0.52 & 2.28 & 2.75 & 0.30 & 0.66 \\
\hline 21 & 0.00 & 0.24 & 20.31 & 36.59 & 18.98 & 17.32 & 1.00 & 2.48 & 2.75 & 0.30 & 0.64 \\
\hline 22 & 0.00 & 0.35 & 21.07 & 29.30 & 20.63 & 18.19 & 4.14 & 2.80 & 3.21 & 0.37 & 0.58 \\
\hline 23 & 0.00 & 1.65 & 24.20 & 36.54 & 17.25 & 11.15 & 4.08 & 2.03 & 2.87 & 0.30 & 0.60 \\
\hline 24 & 0.00 & 0.30 & 21.92 & 37.07 & 20.72 & 14.65 & 0.60 & 2.35 & 2.79 & 0.32 & 0.63 \\
\hline 25 & 0.00 & 0.24 & 20.99 & 35.96 & 16.97 & 17.57 & 2.86 & 2.41 & 2.64 & 0.30 & 0.63 \\
\hline 26 & 0.00 & 0.24 & 19.89 & 37.63 & 16.44 & 19.14 & 1.27 & 2.46 & 2.58 & 0.30 & 0.65 \\
\hline 27 & 0.00 & 0.47 & 21.16 & 35.75 & 14.83 & 21.45 & 0.76 & 2.39 & 2.70 & 0.42 & 0.63 \\
\hline 28 & 0.29 & 0.53 & 20.56 & 31.39 & 20.94 & 20.36 & 0.00 & 2.60 & 2.91 & 0.39 & 0.60 \\
\hline 29 & 0.00 & 0.41 & 18.79 & 38.13 & 14.97 & 20.38 & 1.98 & 2.42 & 2.56 & 0.39 & 0.67 \\
\hline
\end{tabular}

${ }^{a} \mathrm{RAN}=\mathrm{an} /(\mathrm{ab}+\mathrm{an})$
TABLE 11

CIPW Norms of Hole 418A Basalts

\begin{tabular}{|c|c|c|c|c|c|c|c|c|c|c|c|}
\hline Core & Q & OR & $\mathrm{AB}$ & AN & DI & HY & $\mathrm{OL}$ & MT & IL & AP & RAN \\
\hline 1 & 0.00 & 0.47 & & & & & 57 & 2.10 & 2.37 & 0.25 & 0.61 \\
\hline 2 & 00 & .65 & & & .08 & .90 & 8.18 & & 2.36 & .39 & 0.59 \\
\hline 3 & 00 & 35 & .38 & & .53 & 1.70 & 5.54 & 2.26 & 2.07 & 0.28 & 0.64 \\
\hline 4 & 00 & & .89 & 31.78 & 3.18 & 16.46 & 3.48 & 2.26 & 2.01 & 0.44 & 0.62 \\
\hline 5 & 00 & 0.41 & 19.29 & 32.40 & 23.56 & 17.28 & 2.16 & 2.38 & 2.28 & 0.25 & 0.63 \\
\hline 6 & .00 & 0.41 & 0.06 & & 21.60 & 17.89 & 2.57 & 2.20 & 2.15 & 0.25 & 0.63 \\
\hline 7 & 00 & 0.95 & .59 & 35.57 & 18.18 & 1.41 & 6.54 & 2.19 & 2.39 & 0.23 & 0.61 \\
\hline 8 & .00 & 0.30 & .22 & & 19.23 & 18.87 & 0.83 & 2.22 & 2.26 & 0.23 & 0.64 \\
\hline 9 & .00 & 1.30 & .73 & 32.83 & 17.52 & 8.60 & 3.75 & 2.49 & 2.49 & 0.28 & 0.61 \\
\hline 10 & .00 & 0.83 & & & & & & & 2.49 & & 0.64 \\
\hline 11 & 00 & 1.12 & 2 & 40.51 & 14.95 & 11.83 & 6.12 & 2.06 & 2.36 & 0.23 & 0.66 \\
\hline 12 & 00 & 2.66 & & & & & & & 2.36 & & 0.65 \\
\hline 13 & 39 & 0.18 & & & & 14.63 & 0.00 & 2.0 & 2.39 & 0.28 & 0.64 \\
\hline 14 & 25 & 0.59 & .26 & 5 & 15.82 & 20.52 & 0.00 & 1.91 & 2.70 & 0.30 & 0.62 \\
\hline 15 & .00 & 6.15 & & & 17.99 & 8.09 & 7.42 & 2.1 & 2.41 & 0.39 & 0.64 \\
\hline 16 & 00 & 1.54 & & 39 & 9 & 4.67 & 9.91 & 2.3 & 2.41 & 0.28 & 0.66 \\
\hline 17 & & & & & & & & & & & 68 \\
\hline 18 & 00 & 0.1 & & & & & & & 2.07 & & 64 \\
\hline 19 & 00 & & & & & & & & & & 82 \\
\hline 20 & 00 & 1.18 & & 2 & 22 & & 2. & & 1. & & 0.67 \\
\hline 21 & 74 & & & & & & & & & & 0 \\
\hline 22 & & & & & & & 1.7 & 2. & 1. & 0. & 0.66 \\
\hline 23 & & & & & & & & & 2. & & 0.65 \\
\hline 24 & & & & & & & & & & & \\
\hline 25 & 10 & 0.2 & & & 218 & & & & 2. & & 0.65 \\
\hline 0 & & & & & & & & & & & \\
\hline 27 & & & & & & & 0. & & 2. & & 0. \\
\hline 28 & & & & & & & & & & & \\
\hline 29 & & 0. & & & & & & & & & \\
\hline 30 & & & & & & & & & & & \\
\hline 31 & & & & & & & & & & & \\
\hline 32 & 00 & & & & & & & & & & 0. \\
\hline 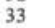 & & & & & & & & & & & \\
\hline 34 & & & & & & & & & 2. & 0. & 0.63 \\
\hline 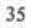 & & & & & & & & & & & \\
\hline 36 & 90 & & & & & & & & & & \\
\hline 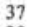 & & & & & & & & & & & \\
\hline 38 & & & & & & & & & & & \\
\hline 39 & 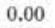 & & & & & & & & & & 0. \\
\hline 40 & & & & & & & & & & & \\
\hline 41 & & & & & & & & & & & 0. \\
\hline 42 & & & & & & & & & & & \\
\hline 43 & & & & & & & & & & 0. & 0. \\
\hline 44 & & & & & & & & & & & \\
\hline 45 & & & & & & & & & & & 0.65 \\
\hline 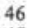 & & & & & & & & & & & \\
\hline 47 & & & & & & & & & & & \\
\hline 48 & & & & & & & & & & & \\
\hline 49 & & & & & & & & & & & \\
\hline 50 & & & & & & & & & & & 0. \\
\hline 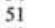 & & & & & & & & & & & \\
\hline 52 & & & & & & & & & & & \\
\hline 67 & & & & & & & & & & & \\
\hline 54 & & & & & & & & & & & \\
\hline 55 & & & & & & & & & & & \\
\hline 56 & & & & & & & & & & & \\
\hline 57 & & & & & & & & & & & \\
\hline 58 & & & & & & & & & & & \\
\hline 59 & & & & & & & & & & & 12 \\
\hline 60 & & & & & & & & & & & \\
\hline 61 & & & & & & & & & & & \\
\hline 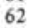 & & & & & & & & & & & \\
\hline 63 & & & & & & & & & & & \\
\hline 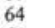 & & & & & & & & & & & \\
\hline 65 & & & & & & & & & & 0. & \\
\hline 66 & & 0. & & & & & & & & & 0.64 \\
\hline 67 & 00 & 0.30 & 18.19 & $\ldots$ & .35 & 21.61 & 0.00 & 2.49 & 2.54 & 0.28 & 0.63 \\
\hline
\end{tabular}

are quartz normative. The normative ratio, $a n /(a b+a n)$, which is a good indication of the average an-content of plagioclase, varies from 0.55 to 0.69 ; the normative color index is between 31.2 and 54.2 per cent.

6) Within the Hole 417D basalt sequence, no significant chemical downhole variations have been observed which can be related to distinct cooling units. Generally, this holds also for the 418A basement section. Among the stratigraphic units established, only Units $6 \mathrm{~b}$ and 13 differ slightly, but significantly, with respect to their $\mathrm{TiO}_{2}, \mathrm{Ni}$, and $\mathrm{Cr}$ concentrations. For all other elements determined, downhole variation does not exceed the variability within stratigraphic units.

7) On the average, basalts from Hole $418 \mathrm{~A}$ are slightly higher in $\mathrm{MgO}$ and $\mathrm{CaO}$ and lower in $\mathrm{TiO}_{2}$, total iron, and 


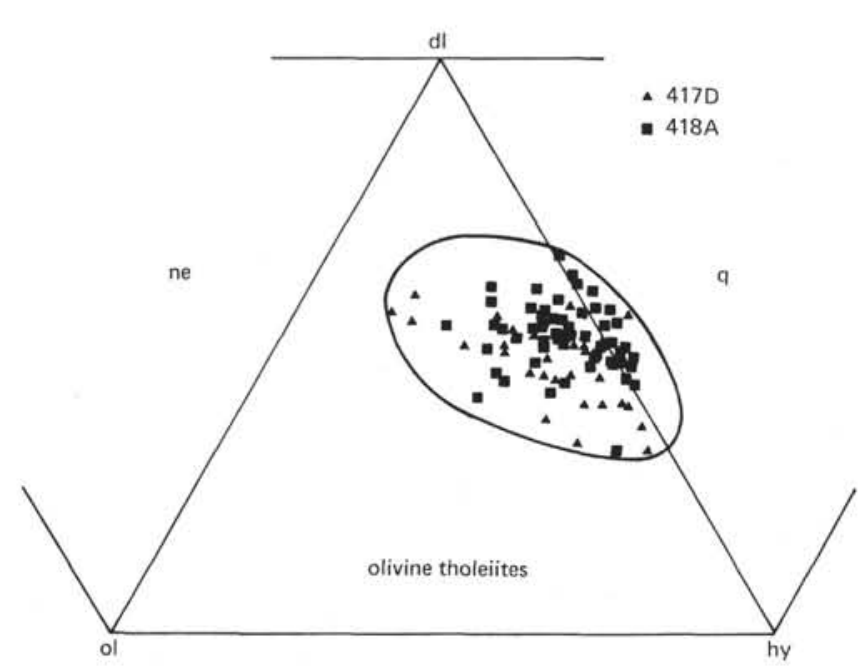

Figure 7. Normative compositions of Hole $417 \mathrm{D}$ and $418 \mathrm{~A}$ basalts plotted on the Tilley-Muir diagram.

$\mathrm{Na}_{2} \mathrm{O}$ than basalts from Hole 417D. These differences in primary major element composition are reflected in minor differences in the trace-element abundances.

8) Since evidence of 15 distinct eruptive events covering a time span of about 100,000 years has been found in the 544-meter section drilled in Hole 418A, it has to be assumed that the magma compositions were governed by more or less constant parameters throughout this time.

These facts have to be taken into consideration when modeling the basalt genesis. In doing so, the averages obtained on Hole 417D and 418A basalts are taken as a close approximation of the chemical composition of the respective magmas.

In general terms, the compositions of basaltic liquids erupted on the ocean floor will be functions of the following: (a) Mineralogical and chemical composition of the peridotitic source material. (b) Degree of partial melting. (c) Extent of fractional crystallization during magma ascent (under high and low pressure conditions). (d) Nature of the crystallizing phases.

Clarification is important as to whether the basalts encountered have been chemically evolved or represent more or less primitive material. As a reliable indicator of the "primitiveness" of these rocks, the ratio $\mathrm{Mg} /\left(\mathrm{Mg}+\mathrm{Fe}^{2+}\right)$ can be applied. It has been demonstrated that basaltic liquids in equilibrium with their peridotitic source material having a $\mathrm{Mg} /\left(\mathrm{Mg}+\mathrm{Fe}^{2+}\right)$ ratio of 0.9 at a degree of partial melting of about 25 per cent will have a $\mathrm{Mg} /\left(\mathrm{Mg}+\mathrm{Fe}^{2+}\right)$ ratio of approximately 0.70 (e.g., Ayuso et al., 1976). Since Hole 417D and 418A basalts show $\mathrm{Mg} /\left(\mathrm{Mg}+\mathrm{Fe}^{2+}\right)$ ratios between 0.50 and 0.63 (see Tables 3 and 8 ), they do not represent truly primitive liquids; these basalts have experienced fractionation of the ferromagnesian phases olivine and/or pyroxene, resulting in a corresponding reduction of the ratio of $\mathrm{Mg}$ to $\left(\mathrm{Mg}+\mathrm{Fe}^{2+}\right)$. As a suitable parameter for distinguishing between a preferentially olivine or a chiefly pyroxene separation, the $\mathrm{Ni}$-concentrations of the basalts can be used. Because $\mathrm{Ni}$ is strongly partitioned into olivine, removal of olivine will result in a pronounced decrease in the $\mathrm{Ni}$ content of the remaining liquid. $\mathrm{Ni}$ concentrations in primitive basaltic melts in equilibrium with their parental peridotites are assumed to be 200 to $300 \mathrm{ppm}$ (Nicholls, 1978). Compared to these values, the Ni contents of Hole 417D and 418A basalts (95 and $101 \mathrm{ppm}$, respectively) show a considerable depletion. Thus, the parallel decrease of both the $\mathrm{Mg} /\left(\mathrm{Mg}+\mathrm{Fe}^{2+}\right)$ ratio and the $\mathrm{Ni}$-abundances suggests that the respective magmas have evolved slightly by removal of (about $15 \%$ ) olivine. Since the distribution coefficients for olivine in the case of all other trace elements (except for $\mathrm{Cr}$ ) are far below unity, olivine fractionation will result in a general enrichment of these elements in the melt without affecting their patterns. This holds for the REE in the same way. The effect of olivine removal on the REE abundances is essentially one of concentrating these elements without changing the initial $\mathrm{La} / \mathrm{Sm}$ e.f.

If pyroxene would have been a major fractionating phase, $\mathrm{Ni}$ decrease should not be as strong as observed. Furthermore, pyroxene fractionation would have effected the composition of the remaining liquid in a mode different from olivine separation: due to different distribution coefficients for the LREE and HREE, pyroxene removal will enhance the LREE to HREE ratio, i.e., affect the REE pattern. Since all basaltic lithologies in the two holes are strongly depleted in LREE, major pyroxene fractionation cannot have occurred during magma development.

Microscopic study of the basalts indicates approximately cotectic crystallization of olivine and plagioclase in a lowpressure environment. But from the data obtained, the degree of plagioclase fractionation is difficult to evaluate. Lack of positive and negative Eu anomalies (Puchelt and Emmermann, 1977) proves that neither a significant plagioclase accumulation nor subtraction can have occurred. Thus, we conclude that the Holes 417D and 418A basalts are derived from magmas evolved by a preferential separation of olivine. The observed small chemical differences between the basalts from Hole 417D and 418A, as well as between Units $6 \mathrm{~b}$ and 13 of Hole $418 \mathrm{~A}$, can be explained by slightly different degrees of low-pressure differentiation. Low-pressure fractionation of small amounts of olivine plus plagioclase will result in a decrease of $\mathrm{MgO}$ and $\mathrm{CaO}$ and an increase of $\mathrm{TiO}_{2}$, total iron, and $\mathrm{Na}_{2} \mathrm{O}$ (as well as in REE) as observed in the Hole 417D basalts.

The variability measured within stratigraphic units can be likewise related to differentiation and is most probably caused by processing during emplacement, e.g., crystal settling, flow differentiation, etc. (Figure 8).

Basalts recovered from the Bermuda Triangle sites are products of Cretaceous mid-ocean ridge volcanism and were most probably generated some 110 m.y.B.P. at the ancestral Mid-Atlantic Ridge (MAR) at about $18^{\circ} \mathrm{N}$. To trace secular variation in basalt composition, it is tempting to compare these basalts to older and younger basalts which originated within the same portion of the MAR. For this purpose, chemical data from Leg 46 and Leg 11 basalts can be used. Leg 46 basalts (drilled at about $22^{\circ} \mathrm{N}$ some $160 \mathrm{~km}$ east of the MAR), with an inferred age of approximately 13 m.y.B.P., may be regarded as modern equivalents of the basalts drilled at Sites 417 and 418. Leg 11 (Site 100) basalts $(\sim 150$ m.y.B.P.) represent predecessors of Hole 417D and 418A basalts and originated shortly after the opening of the North Atlantic at the ancestral MAR at about 

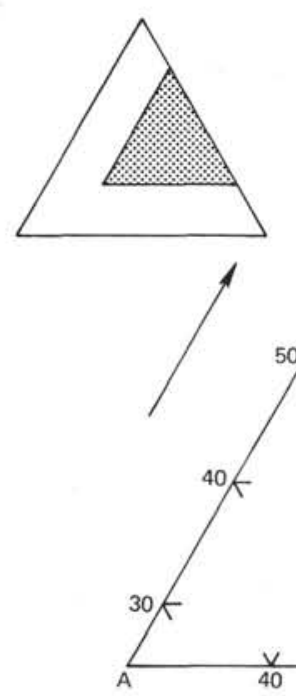

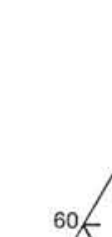

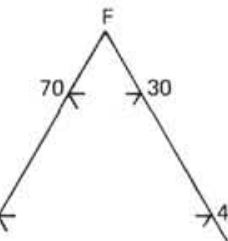

40
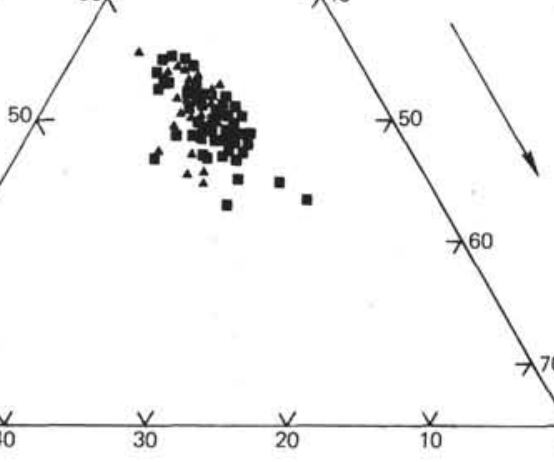

$\frac{v}{20}$

\section{(1)}

Figure 8. AFM plot of Hole $417 D$ and 418 A basalts.

$16^{\circ} \mathrm{N}$. In this comparison, data from Triassic dolerites from the southern Appalachian Mountains (Georgia) of Eastern North America (ENA) will also be included, because these rocks are to be regarded as the first manifestations of a basaltic magmatism related to the opening of the Atlantic. If the eastern coast of North America is projected back to the MAR along accepted crustal-spreading "'flow-lines,", what is now Georgia projects to about $18^{\circ} \mathrm{N}$ (Bryan et al., 1977).

In their major element chemistry, ENA dolerites and basalts from Legs 11, 51-53, and 46 are very similar, all being olivine tholeiites. Systematic differences exist in trace-element abundances and are especially pronounced with the LIL elements (e.g., $\mathrm{K}, \mathrm{Li}, \mathrm{Rb}, \mathrm{Ba}, \mathrm{Zr}$, and LREE). This group of elements plays an important role in modeling the basalt genesis, because they preferentially enter the initial melt. If there is no supply of new mantle material, each successive partial melting event will provide magmas which are increasingly depleted in LIL elements. A pronounced evolutionary trend is obvious when one compares the abundances of selected LIL elements in these basalts of a different age. For comparison purposes, the concentrations of the elements selected were normalized against the respective values obtained on Leg 46 basalts (Emmermann and Puchelt, 1979), because they represent the latest stages in this development. As seen from Figure 9, the Triassic dolerites from ENA are clearly enriched in K and LREE, whereas these elements are strongly depleted in the Jurassic and Cretaceous ocean-floor basalts. Assuming that at least the oldest (i.e., ENA and Leg 11) basaltic melts originated within the same mantle volume and that neither have been affected by alteration or continental contamination, the distinct depletion of LIL elements in the Jurassic basalts can be explained by remelting of a "pre-extracted" mantle material. The pre-extracted fraction might be represented by the Triassic basaltic rocks. The increase of LIL elements in the younger basalts suggests that following this event, the basalt source region was gradually replaced by undepleted material. From the data presently available, it cannot be decided whether this process was unidirectional or cyclic.

\section{CONCLUSIONS}

The volcanic piles drilled in Holes 417D and 418A are products of Cretaceous mid-ocean ridge magmatism and were built up in a number of intermittent extrusive events within a time span of approximately 100,000 years. During all this time, magmas of rather uniform compositions erupted, indicating that the processes of magma generation and development were governed by constant parameters. All basalts recovered are low-K tholeiites and closely resemble modern mid-ocean ridge basalts. A typical feature is their very low content in LIL elements and, especially, their strong depletion in LREE. From the $\mathrm{Mg} /\left(\mathrm{Mg}+\mathrm{Fe}^{2+}\right)$ ratios and $\mathrm{Ni}$ abundances of the basalts, it can be deduced that the magmas do not represent truly primary liquids, but have been evolved mainly by removal of olivine. The minor differences in the average compositions between Hole 417D and $418 \mathrm{~A}$ basalts are explained by a slightly different degree of low-pressure fractionation of olivine and plagioclase. From the REE patterns and the mode of differentiation, it is concluded that the basalts recovered from the Bermuda Triangle sites originated from a peridotitic mantle source which was already depleted in LIL elements by previous melting event(s).

\section{ACKNOWLEDGMENTS}

This research was supported by the "Deutsche Forschungsgemeinschaft" under Project Pu-15/19 and Em-23/2. The samples were irradiated for INAA in the Karlsruhe reactor FR 2 with financial support by the Kernforschungszentrum Karlsruhe. U. Kramar made INAA determinations with equipment provided by the Ministerium für Forschung und Technologie, FRG. We thank all individuals and institutions who contributed to this investigation. The manuscript was critically reviewed by W.B. Bryan, who made valuable suggestions.

\section{REFERENCES}

Ayuso, R.A., Bence, A.E., and Taylor, S.R., 1976. Upper Jurassic tholeiitic basalts from DSDP Leg 11: J. Geophys. Res., v. 81 , p. $4305-4325$.

Bryan, W.B., Frey, F.A., and Thompson, G., 1977. Oldest Atlantic seafloor, Contrib. Mineral. Petrol., v. 64, p. 223-242.

Byerly, G.R. and Wright, T.L., 1978. Origin of major element chemical trends in DSDP Leg 37 basalts, Mid-Atlantic Ridge, J. Volcanol. Therm. Res., v. 3, p. 229-279.

Dietrich, V., Emmermann, R., Keller, J., and Puchelt, H., 1977. Tholeiitic basalts from the Tyrrhenian sea floor, Earth Planet. Sci. Lett., v. 36, p. 285-296.

Donnelly, T.W., Francheteau, J., Salisbury, M., Kelts, K., Swift, S.A., Borella, P.E., Bleil, U., Smith, B.M., Juteau, T., Rusinov, V., Linton, J.M., Ui, T., Orr, W.N., and Gartner, S., 1977. Mid-Ocean-Ridge in the Cretaceous, Geotimes, v. 22, p. 21-23.

Emmermann, R., and Puchelt, H., 1979. Abundances of rare earths and other trace elements in Leg 46 basalts (DSDP). In Dmitriev, L., Heirtzler, J., et al., Initial Reports of the Deep Sea Drilling Project, v. 46: Washington (U.S. Government Printing Office), p. 241-246.

Flower, M. and Salisbury, M., et al., 1977. Cretaceous crust sought, Geotimes, no. 9, p. 20-22.

Masuda, A., 1975. Abundances of monoisotopic REE, consistent with the Leedey chondrite value, Geochim. J., v. 9, p. 183184.

Nicholls, J.A., 1978. Primary basaltic magmas for the pre-caldera volcanic rocks of Santorini, Acta of the 2 nd International Scientific Congress on the Volcano of Thera, Athens 1978. 


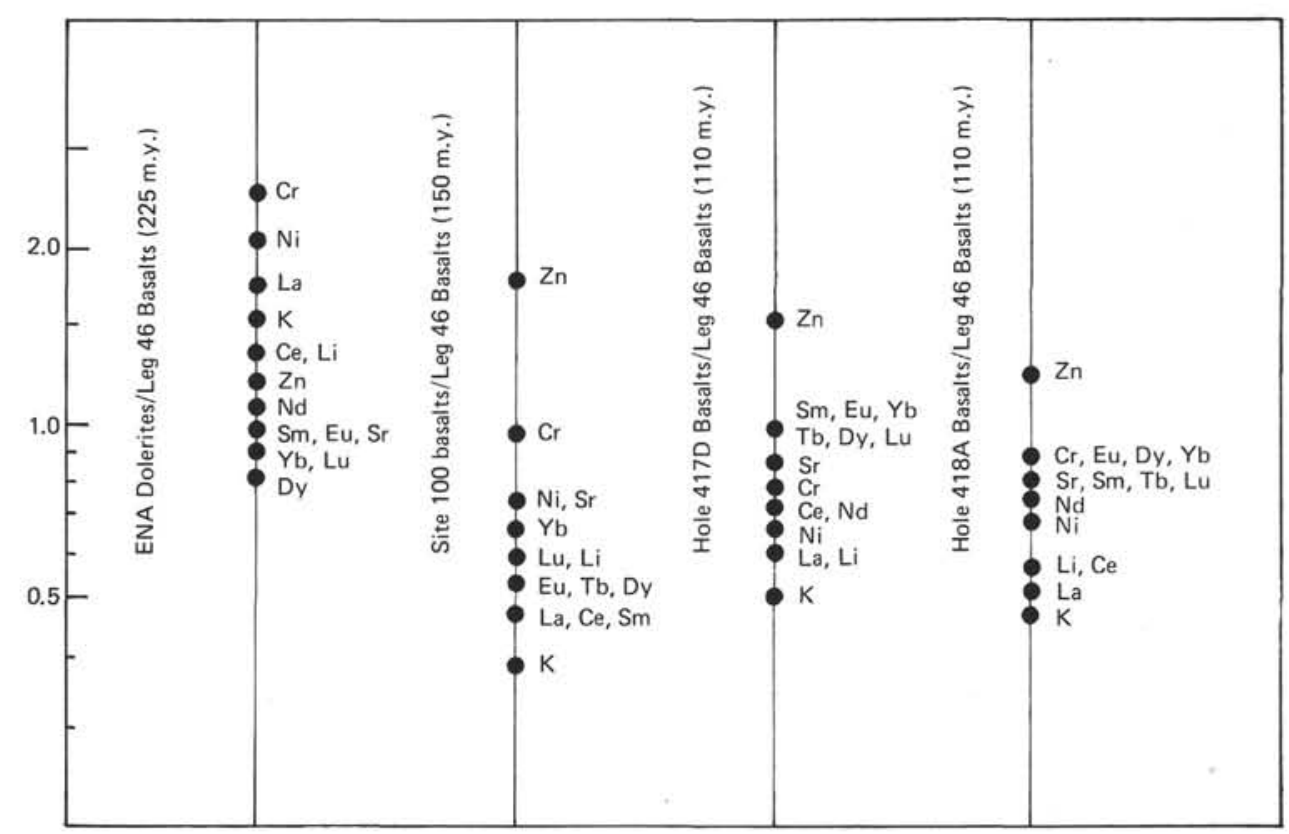

Figure 9. Averages of selected elements in ENA dolerites, Leg 11, and Leg 51-53 basalts normalized against the respective values in Leg 46 basalts.

Puchelt, H. and Emmermann, R., 1977. REE characteristics of Ocean Floor Basalts from the MAR $37^{\circ} \mathrm{N}$ (Leg 37, DSDP), Contrib. Mineral. Petrol., v. 62, p. 43-52.

Puchelt, H., Emmermann, R., and Kramar, U., 1976. The bearing of trace element distribution patterns of deep-sea basalts for the interpretation of their genesis, J. Radioanalyt. Chem., v. 2, p. 1049-1056.

Wedepohl, K.H., 1975.The contribution of chemical data to assumptions about the origin of magmas from the mantle, Fortschr. Miner., v, 52, p. 141-172. 